\title{
Preparative two dimensional separations involving Liquid-Liquid Chromatography
}

\author{
Léa Marlot and Karine Faure \\ Université de Lyon, CNRS, Université Claude Bernard Lyon 1, ENS de Lyon, Institut des Sciences \\ Analytiques, UMR 5280, 5 rue de la Doua, F-69100 VILLEURBANNE, France \\ karine.faure@isa-lyon.fr
}

\section{Abstract}

The increasing performances of analytical techniques, especially two-dimensional liquid chromatography hyphenated with highly sensitive detectors, allow discovery of new targeted compounds in highly complex samples, whether biomarkers in environmental effluents, natural metabolomes in natural products or unknown impurities in synthetic chemical process. While structural identification can usually be achieved through mass spectrometry and databases, it can be useful to confirm the potential structure via NMR analysis and/or to obtain standard reference material for quantitative purposes, incentive for the production of $\mu \mathrm{g}$ to $\mathrm{mg}$ of new target compounds. Hence, preparative chromatography, which was initially run for large-scale production, is now facing new challenges, with small volumes of highly complex samples to deal with. Unfortunately, one dimensional chromatographic methods exhibit limited performances when targets are minor compounds in a complex matrix. Moreover, for process intensification or limited amount of sample, it is now a priority to be able to isolate multiple components with the largest yield possible using as few purification steps as possible. With these specifications in mind, a comprehensive multidimensional chromatographic method for preparative purposes is becoming appealing.

Liquid-liquid chromatography, also known as countercurrent chromatography or centrifugal partition chromatography, is a technique with a proven high loading capacity, working with multiple choice of solvent systems thus a large range of polarity and selectivity. The liquid nature of the stationary phase prevents sample denaturation, irreversible adsorption resulting in high sample recovery. All these qualities make the LLC technique highly receivable as a first dimension in a 2D strategy, whether combined with another LLC method or with a more conventional LC method. In this review, both hyphenations are investigated from an instrumentation point of view, detailing systems selection methodology and two dimensional development strategy. Preliminarily, the specific LLC instrumentation is briefly summarized in this review and the nomenclature of two dimensional separation as well as the associated criteria are clearly explained and defined.

\section{Introduction}

In the late 1960s, Ito invented countercurrent chromatography (CCC) [1], a liquid chromatography technique which originality remains in the liquid nature of the stationary phase. The name countercurrent chromatography was derived by analogy to Craig's countercurrent distribution system implemented in 1940s [2-4]. This chromatographic concept led to two different instrumental developments. Ito pursued the development of the original hydrodynamic CCC apparatus (Figure 1a) $[5,6]$ whereas Nunogaki focus on a hydrostatic version (Figure 1b) called centrifugal partition chromatography (CPC) $[6,7]$. Both technologies are based on the use of a liquid stationary phase and 
a liquid mobile phase between which the solutes partition, their difference being only the way the centrifugal field is applied. Hence, for the sake of the reader's understanding, the global technique is called Liquid-Liquid Chromatography (LLC) in this review, independently of the instrument used.

In hydrodynamic CCC instruments, the stationary phase is retained by centrifugal forces in a planetary spinning coil while in hydrostatic CPC, the stationary phase retention is assisted by the use of tiny oblong or rectangular chambers connected by capillary ducts rotating around a single axis (Figure 1). It should be clear that the basic theory and concepts are the same for both apparatus. Only the instrumentation and the way the phases intermingle differ.

\section{[here Figure 1]}

The main specifications of LLC are related to the liquid nature of the stationary phase $[6,8]$. Compared to LC, the LLC technique allows a larger sample loading capacity, with a relatively low number of chromatographic plates $[8,9]$, which classify it mainly as a preparative technique. Moreover, many solvent systems are available and overlay a wide range of polarity of compounds [10]. Both phases of the solvent systems can be used as mobile or stationary phase, which drastically decreases the cost of the columns as compared to conventional preparative LC columns.

The most important advantage is the total sample recovery. Since no supporting matrix is employed, the separation is not distorted by solute adsorption or catalytic change encountered with solid supports. Moreover, the solutes remaining in the column can be easily recovered by extrusion of the liquid stationary phase.

Liquid-Liquid Chromatography, LLC, has progressed both instrumentally and methodologically in last decades, to be an effective technique for isolation and purification. It has found a large audience in the natural products science area [4, 9, 11-14], which represents a rich source of biologically active compounds, widely used for medical purposes for centuries, driving nowadays pharmaceutical development. More recently, innovative coupling strategies have been carried out in order to develop the separation power and the detection potential. In 2014, Michel et al. have described these coupling strategies in a review [15] highlighting the online coupling strategy in an instrumental point of view. It includes the online hyphenation of LLC with different detectors such as MS and NMR but also with other chromatographic techniques such as LLC, HPLC or GC, with an analytical goal. In 2016, Xie et al. published a review concerning the combinations of prep-HPLC with other chromatographic techniques applied to traditional Chinese medicine [16] mentioning some online and offline couplings of LLC with LC at preparative scale.

The goal of the present review is to provide an overview of the two dimensionaltwo dimensional preparative separations involving LLC technique. Under our investigation, 12 publications were found involving the hyphenation of the LLC technique with another LLC apparatus in online and offline modes since 2002 (Table 1). Of these 12 applications, only one application was implemented at analytical scale with a view for a preparative scale transfer. The other applications were implemented directly at preparative scale in order to recover the compounds of interest.

Since 2007, 21 publications were found involving the hyphenation of the LLC technique with LC in two dimensionaltwo dimensional separations in online and offline modes (Table 2). Of these 21 publications, three applications were implemented at analytical scale with a possible preparative scale transfer. The other applications were implemented at preparative scale.

Some hyphenations of LLC with GC were found in literature as two dimensionaltwo dimensional separations $[17,18]$ but these hyphenations are outside the research framework presented here due to the obstacle of preparative scale transfer.

\section{Nomenclature and criteria for two dimensional separations}


In the liquid chromatography field, several studies have been published in order to clearly define the term of two dimensional separation and the associated notions. This is not the case in LLC field and several terms are incorrectly employed in LLC publications without any theoretical background. In 2007 and 2008, Stoll et al. [19], Dugo et al. [20] and Guiochon et al. [21] published three studies introducing the theory and applications of multidimensional liquid chromatography. In 2009, another review has been published by François et al. [22] focusing on the fundamental aspects of multidimensional liquid chromatography. From these reviews, the term "two-dimensional liquid chromatography" refers to the technique in which two independent liquid phase separation systems, noted ${ }^{1} \mathrm{D}$ and ${ }^{2} \mathrm{D}$ for first and second dimensions, are applied to the sample. The largest benefit of combining columns in two dimensional configuration is the improvement of peak capacity, which is reflected in the reduction of component overlap. When the selected separation mechanisms provide completely different selectivities, the advantages of the two dimensional approach are fully exploited, since the potentiality of co-eluting sample components in both dimensions are severely minimized. Separation potential quickly increases as the correlation between the retention mechanisms decreases. In a preparative sense, this resolution improvement relates to the purity that can be expected.

Two distinctions have to be done dependent on the way the effluent is transferred from the first dimension column to the second dimension column. The two dimensional separation can be implemented in online or in offline mode. In offline two dimensional separations, the execution is very simple since the fractions of the first dimension effluent are collected, after which they are concentrated if necessary and re-injected on the second column. However, this method can be time consuming, difficult to automate and reproduce, susceptible to sample loss and contamination. In online mode, the columns in the two dimensions are connected via an appropriate interface. This approach is more difficult to implement and to operate, however, faster and more reproducible.

Two dimensional separations can be done by transferring either only the interesting portion of the first dimension, this is referred to as chromatography in heart-cut mode, or by sequentially transferring the entirety of the first dimension effluent, in many small aliquots, to the second dimension; this is known as comprehensive chromatography. In 2012, Schoenmakers team published an update concerning the nomenclature and the conventions in comprehensive multidimensional chromatography [23]. In this update, the comprehensiveness is clearly defined and the term "comprehensive analysis" does not mean "analyzing everything". Three criteria were established: 1- Every part of the sample is subjected to two different (independent) separations; 2- Equal percentages (either 100\% or lower) of all sample components pass through both columns and eventually reach the detector; 3 - The separation (resolution) obtained in the first dimension is essentially maintained.

In a preparative view, these three criteria can be applied, keeping in mind that the percentage of transfer will have a huge influence on the final yield of the product.

In two dimensional LC, numerous approaches are available to evaluate the separation power of the column combination. In the literature, the measurement of the peak capacity can be found as well as the measurement of the orthogonality between dimensions. Schoenmakers [23] has defined the orthogonality as two instrumental dimensions that possess different mechanisms of separation, where elution times in the two dimensions can be treated as statistically independent. Orthogonal separation mechanisms depends on the sample and the specific physical-chemical interactions that the solutes have with the systems. The orthogonality between two systems exists since the systems provide different selectivities which are the abilities of the chromatographic systems to chemically distinguish the sample components. The higher the orthogonality, the better the separation power.

In order to evaluate the orthogonality of a two dimensional separation, the retention times of the compounds in first and second dimensions have to be plotted on a two-dimensional map (as presented in Figure 2). For LLC two dimensional separations, the partition coefficients $\mathrm{Kd}$ values (and the 
retention factors $\mathrm{k}$ values for HPLC separations) can be plotted instead of retention times in order to eliminate the variations of the column dimensions and operating conditions. Obviously, if the separations are strongly correlated (Figure $2 \mathrm{a}$ and $\mathrm{b}$ ), critical pairs in a dimension will remain close in the second dimension and hence the loading capacity in preparative chromatography will remain low. At the opposite, if no correlation exists between the two dimensions (Figure $2 \mathrm{~d}$ ), it is possible to produce highly concentrated fractions of overlapping compounds using the first dimension knowing that the second dimension will afterwards separate the coeluting compounds.

Several approaches can be found in the literature to measure and optimize orthogonality. These approaches can be thermodynamic approaches which only consider the retention data of the compounds or can be thermodynamic and kinetic models which also include peak broadening.

The bin-counting method proposed by Gilar et al. $[24,25]$ is strongly affected by the bins width decision that the user makes to divide the separation space .

Slonecker et al. proposed to described the orthogonality by information theory [26]. Such mutual information includes peaks which cluster along the upward diagonal of the separation space. The proportion of mutual information compared to the total separation space is expected as low as possible. The limitation of this method is the reliance on the assumption that peaks only cluster along the upward diagonal.

Liu et al. developed a geometrical approach based on the factor analysis [27]. This method shares the same limitation than the previous one due to the assumption that peaks only cluster along the upward diagonal.

In the convex-hull approach [28], the retention space of the compounds is defined by an area which is delimited by the most distant points on the two dimensional representation. The orthogonality is directly linked to this area. Empty parts of the separation space may introduce bias on orthogonality evaluation.

The nearest-neighbor distance approach has been proposed by Nowik et al. [29, 30] in order to calculate the distance from a given peak to its closest neighbor. The harmonic mean can be related to the degree of the clustering. This method is however highly dependent on the number of the peaks within the separation space.

The asterisk equations approach proposed by Schoenmakers [31] is based on the normalized retention data of the compounds in each dimension. Despite the easy use of this method, the calculation of the orthogonality is based on normalized data which do not represent the effective total separation space.

While this list may not be exhaustive, these proposed methods for measuring chromatographic orthogonality have their limitations and should be compared and discussed. But when dealing with preparative chromatography, these notions are not taken into account so far and to the best of our knowledge, orthogonality evaluation has never been applied to the LLC field, not to mention optimization. In this review, we will focus on the first pre-requisite, i.e. two-independent separation mechanism for a preliminary evaluation of orthogonality.

For this purpose, two parameters can be taken into account. The first parameter is the global correlation coefficient of the 2D-plot $r^{2}$. Orthogonal separation mechanisms by definition should not be correlated, thus the peaks should spread throughout the separation space. As shown in the Figure 2 , when the correlation coefficient is equal to 1 (Figure 2a), the chromatographic interactions endured by the solutes are similar which means absence of orthogonality between the systems. As explained by Stoll et al. [19] and presented in Figure 2c, the 2D-plot correlation coefficient $r^{2}$ can be low without an important utilization of the two dimensional separation space. Thus, to ensure a higher orthogonality of the two dimensional separation, which will relate to better resolution and hence better purity in preparative schemes, the coefficient correlation $r^{2}$ has to be close to 0 and the separation space has to be the largest as possible (Figure 2d). Moreover, in preparative chromatography, a special attention should be brought to clusters (Figure 2c). Indeed, while during method development, the compounds may appear resolved with sufficient purity using 2D separation, 
the production aim, even set at few $\mathrm{mg}$, provide the incentive for concentration and loading, which inevitably results in peak broadening and overlapping.

[here Figure 2]

\section{Two dimensional LLC-LLC separations}

From 1998 until 2015, the concept of two dimensional LLC-LLC separation have only been investigated on CCC apparatus, meaning coil-based instruments. Table 1 reports the publications found under our investigations, classified according to the selected transfer strategy, either online or offline. The interface, the fractions volume, and the instrumentation are described for comparison. The solutes are indicated in the references but are not the main purpose of the discussion here.

These two-dimensional separations have mainly been developed for preparative purposes of selected solutes, so only heart-cut mode was carried out, meaning that a limited number of fractions from the first dimension were transferred to the second dimension. Up to date, $17 \%$ of the applications are implemented in offline mode, thereby $83 \%$ of the applications are implemented in online mode. Compared to the offline mode, the online hyphenation needs a special equipment and technical skills. The fraction of interest from the first dimensional LLC has to be transferred in the second dimensional LLC with a special interface such as a switching valve, a sample loop or a solid-phase interface.

\section{1- Switching valve interface}

The very first online LLC-LLC separation using a switching valve was performed by Ito's group in 1998 [32] where only one fraction from the first dimension was directly introduced to the second dimension. As shown in Figure 3 , the switching valve directly connects the ${ }^{1} D$ eluent to the ${ }^{2} D$ column. The second column was equilibrated and kept in rotation, while the elution in the first column was running with UV detection and fraction collection. When the peak of interest was eluting out the first column, the valve was switched to introduce the ${ }^{1} \mathrm{D}$ eluent directly to the second LLC column. After peak transfer, the valve was switched back to loading position, the pump of the second instrument was started and the elution of the selected targets could run in the ${ }^{2} \mathrm{D}$ column while the ${ }^{1} \mathrm{D}$ elution was back in process. In the cited reference, the solvent system used in the secondary column was the exact same than the one used in the primary column. Indeed, Ito developed this instrumentation in order to lengthen the column to increase resolution on selected parts of the chromatogram, which implies using the same solvent system in first and second coils. This application cannot then be considered as a two dimensional separation, since the same separation mechanism is implemented in both coils. However, this instrumentation set the stage for further 2D CCC development.

\section{[here Figure 3]}

If the first objective was to lengthen the column with this instrumentation, Ito's group developed then three others applications in 2002, 2005 and 2006 using the same HSCCC apparatus and the same interface for 2D separations [33-35]. In each paper, only one fraction of interest was introduced in the second LLC column (heart-cut mode). As a part of the ${ }^{1} \mathrm{D}$ eluant is directly carried into the second LLC column, the second solvent system has to be stable towards the ${ }^{1} \mathrm{D}$ mobile phase, to avoid hydrodynamic equilibrium perturbation and 2D stationary phase loss. In 2002 and 2006, Ito's group used the very same solvents for the two columns or slightly changed the composition of the ${ }^{2} D$ solvent system [33, 35], which can be considered once again as a lengthening of the column. In 2005, however, the $n$-hexane/dichloromethane solvent in first dimension was switch to chloroform in the second dimension solvent system which apparently did not perturb the hydrodynamic equilibrium [34]. It can be considered with these two slightly different columns, hence selectivity, that 2D-CCC emerged. With this direct introduction strategy, the solvent compatibility is the main issue and only slight changes of 
composition are allowed. In terms of orthogonality, this strategy can only provide a weak improvement of the selectivity and peak separation.

More recently, Englert et al. [36] implemented online two dimensional separations using the same switching valve strategy. Englert et al. used a second dimension to slightly change the selectivity on a critical pair. The Kd values of the compounds in the cited solvent systems were estimated, thus the 2D orthogonality representation can be illustrated as shown in Figure 4 . The correlation coefficient is found at 0.875 . This coefficient being close to 1 , the separation mechanisms of the two dimensions are considered highly similar and consequently the orthogonality between the two dimensions is low. Changing $n$-hexane by cyclohexane did not bring selectivity as much as expected and the similar separation mechanisms limited separation improvements.

[here Figure 4]

The main strategy developed in the works using a direct transfer of fractions from one column to another was to establish a first LLC separation with the best peak resolution and selectivity in a reasonable elution time and then to send only the unresolved compounds, usually one critical pair, to the second dimension for further separation. Unfortunately, because of the liquid nature of the ${ }^{2} D$ stationary phase, the transfer of a large volume of ${ }^{1} D$ mobile phase impacts the ${ }^{2} D$ column stability and drastically reduces the choice of the ${ }^{2} \mathrm{D}$ solvent system. With this kind of interface, the two dimensional potential of the separation cannot be fully exploited.

\section{2- Sample loop interface}

As in 2D-LC, a sample loop can be used as interface. In 2007, Pan's group decided to implement a two dimensional separation using a sample loop for a precise control of the transferred volume. A 146-ml HSCCC column was used as first dimension and one 1500-ml CCC coil was used as second dimension. As shown in Figure 5, a 50-ml sample loop was used as interface with three 3-port valves. In a first step, the ${ }^{2} \mathrm{D}$ column was equilibrated while the injection of the sample in the first dimension was made. The valves were switched to introduce the peak of interest to the sample loop. When the cut was over, the valves were switched back to transfer the loop content in the second LLC column and the ${ }^{2} \mathrm{D}$ elution ran while the elution in the first dimension was still in process. With this interface, the solvent compatibility problem is somehow minimized as the transferred volume represents only $3 \%$ of the ${ }^{2} \mathrm{D}$ column volume. However, precautions are still taken in the solvent system selection to avoid hydrodynamic equilibrium perturbation. Indeed, it can be seen that Pan's group selected the same solvents for the two columns ( $n$-hexane-ethyl acetate-methanol-water) and just changed their respective percentages (1:5:1:5 in ${ }^{1} D$ to $3: 5: 3: 5$ in $\left.{ }^{2} D\right)$. As expected, this choice limited the equilibrium perturbation, but kept the orthogonality low.

In the following years, the same group used this 2D instrumentation with loop interface in various coil configurations, transferring to the second dimension from $6 \%$ column volume [38] to $14 \%$ column volume [39].

[here Figure 5]

As for the previous interface, it is regretful that no global two dimensional strategy was employed for the selection of solvent systems. In the three publications, one or two compounds have undergone the full two dimensional separation so the analysis of an orthogonality plot is not relevant.

\section{3- Solid-phase trapping column interface}


In 2010, Pan's group decided to implement a solid-phase trapping interface in the online two dimensional LLC-LLC instrumentation [40]. For this hyphenation, one 6-port switching valve, one 4port switching valve and one trapping column were used with a make-up pump as presented in Figure 6. This configuration using two columns (coils) inside a single LLC instrument was named "multichannel". By switching valves, the peak of interest from the first dimension was loaded in the trapping column (Oasis HLB sorbent) after water was added with a make-up pump to increase analyte retention on the trap. Nitrogen was flushed to dry the column and remove any residual solvents. Then the trapped analytes were back-flushed to the second LLC column with the ${ }^{2} \mathrm{D}$ mobile phase, while the elution was still running in first dimension. The serious advantages of this interface are the avoiding of a large volume injection on the second column by the total elimination of the ${ }^{1} \mathrm{D}$ mobile phase and the focusing effect of analytes before the second separation. The water and nitrogen flush ensure that absolutely no residual solvent is sent from one dimension to the other. This extra precaution may be too harsh on some analytes, which can be either degraded or irreversibly adsorbed on the trap as mentioned by Pan $[40,41]$.

[here Figure 6]

In 2011, Pan's group [41] incremented their 2D instrumentation by setting a solid-phase trapping interface with two trapping columns allowing two fractions to be sent to the second dimension. Once again, the trapping columns were filled with Oasis HLB sorbent type. In this work, two very different solvent systems were used: $n$-hexane/ethyl acetate/methanol/water in first dimension and methyl tert-butyl ether/acetonitrile/water in second dimension. This major change should provide very different selectivities and maximize orthogonality. Unfortunately, as seen on Figure 7, over the four compounds that underwent the two dimensional separation, only the first one seems to be affected by this switch. The overall correlation coefficient is 0.4317 which is globally low, but if we focus on compounds 2, 3 and 4, then it is visible that correlation is high and separation mechanisms are very similar. The improvement brought by the use of a second dimension is alas not obvious for these compounds.

[here Figure 7]

This solid-phase trapping interface allows the use of two chemically different biphasic solvent systems without any phase disturbance. Thus the separation mechanisms can be fully independent and the selectivity and orthogonality can be improved. Unfortunately, in Pan's work, the solvent systems were set up with the same solvent nature in different percentages. While the instrumentation made it possible to overcome a challenge, the selection of the solvent systems in the cited examples was not done as to provide completely different selectivities.

\section{4- Offline mode}

To circumvent the difficulties linked to the transfer of fractions to the second dimension, and to be able to use conventional instruments in a timely manner, it is easier to physically collect fractions eluting from the first separation, eventually applying a sample prep, before injection in the second dimension. This is the case for $17 \%$ of the papers dealing so far with two dimensional LLC-LLC separations.

The first uses of the offline mode for LLC-LLC were implemented by Chen's group in 2013 [42-44] and the objective was to lengthen the column by using the same solvent system in first and second dimension. The recycling mode was also used for this purpose. This mode allowed to prolong the LLC 
separation course to improve the separation factor. These applications are then not considered as two dimensional separations since the very same separation mechanism was set in the two columns.

Currently Chen's group is very active in LLC-LLC two dimensional separations in offline mode $[45,46]$. In the two publications, the sample has been injected in the first LLC dimension then the fractions of interest were collected and the solvents were evaporated before dissolution of the compounds of interest in the mobile phase of the second dimension. In this mode, several fractions could be sent to the second dimension. The transfer is convenient and can be applied more widely. However it needs more manual steps and manipulations which can introduce errors and contaminations. In the two examples shown by Chen et al., the strategy to implement the two dimensional separation was a stepby-step strategy. The first biphasic solvent system allowed an efficient separation with suitable Kd values with a satisfactory separation time. The second biphasic system was chosen to improve the selectivity between the unseparated peaks from the first dimension. Since the solvent of the ${ }^{1} D$ fraction of interest was eliminated by evaporation, the solvent system in second dimension could be completely different with an important selectivity improvement. In the first publication, targeting flavonoids from tartary buckwheat, two chemically different solvent systems were used: $n$ hexane/ethyl acetate/methanol/water in the first dimension and ethyl acetate/n-butanol/water in the second dimension. Unfortunately, as seen on Figure 8a, these two different solvent systems exhibit almost the same interactions with the solutes so separation mechanisms are correlated and orthogonality of the 2D separation is really low. The selectivity is identical and one can question the need of two separations in such conditions.

On the contrary, for the second example from Chen's group, targeting antioxidants from Semen cassia [46], the correlation coefficient is close to 0 (Figure 8b) and the two dimensional separation space is largely occupied. Indeed, in the second dimension, an aqueous copper solution was added to the same solvent system than in first dimension, to implement complexation which completely modifies the solutes retention. This induces a higher orthogonality and a better separation efficiency.

\section{[here Figure 8]}

This issue of solvent system comparison according to their different selectivities towards the targeted compounds is actually scarcely discussed. In 2015, das Neves Costa et al. published a new approach concerning the solvent system selectivities [47]. For a given application, two suitable solvent systems are selected and the Kd values of the compounds of interest in the two selected solvent systems are plotted on a 2D-plot with their peak widths. This is the only representation of an experimental 2D-plot in the LLC literature so far (Figure 9). It highlights the various selectivity correlations depending on the compound chemical nature, for example compounds 1-4 flavonoid glycosides. Then each critical pair selectivity is reported on a scale range helping in the selection of one system or the other according to the selected target. While this approach is not use to develop a 2D methodology, i.e, using both solvent systems for the multi-targeted sample purification, this point of view is very appealing to help the selection of the best solvent system for a specific objective.

[here Figure 9]

The combination of 2D LLC-LLC have been implemented to improve the separation as compared to only one dimension. While the improvement is sometimes only due to a longer column, an increase in selectivity on a critical pair can most of the time make the difference. It is however regretful that the orthogonal strategy is only exploited at the margin. Online interface has focused a lot of instrumental effort and now with the use of solid-phase interface it can allow real two dimensional strategy for the selection of solvent systems. Offline combination require time-consuming fraction handling but can lead to the same results without the financial investment. 
The difference between a heart-cutting multi-dimensional chromatographic technique and a comprehensive one is that the first enables the re-injection of a limited number of multi-component effluent fractions from a first to a second column, while in the comprehensive mode the entire sample is subjected to separation in both dimensions. So far in the literature, only heart-cutting strategies have been developed using LLC-LLC. In the case of more complex samples (over 50 compounds as found in natural products for example), a better orthogonality could definitely improve peak capacity and in a comprehensive strategy, allow the purification of a large number of compounds.

[here Table 1]

\section{Two dimensional LLC-HPLC separations}

The major issue restricting the use of one-dimensional solid-phase liquid chromatography (prepLC) is the limited loading capacity that is due to the non-linear adsorption isotherms on solid stationary phase. On the other hand, the chromatographic efficiency of particle-based prepLC results in low band broadening and hence better resolution of closely retained compounds. Liquid-liquid chromatography (either countercurrent chromatography, or centrifugal partition chromatography) is based on the use of a liquid stationary phase. This allows the injection of large amounts of complex sample without the major overloading issues encountered in LC. This LLC fractionation according to partition coefficients provides to the ${ }^{2} \mathrm{D}$ LC simplified pools of molecules and hence, concentrated amounts of minor compounds can be injected without competition with major compounds.

Up to date, $67 \%$ of the applications were made offline. Thereby, $33 \%$ of the applications were made online using different interfaces. The separations were performed in heart-cut mode but also in a comprehensive mode. Both LLC instrument types, namely CCC and CPC, were used for this purpose.

\section{1- Offline mode}

To carry out an offline two dimensional LLC-HPLC separation, two ways were used in order to manage the solvent compatibility for the transfer of the LLC fractions to HPLC dimension.

In eight publications [48-55], the fractions of interest from the LLC dimension were collected and the solvents were evaporated and solubilized in the ${ }^{2} \mathrm{D}$ mobile phase. However, sample stability upon dryness and secondary dissolution in the ${ }^{2} \mathrm{D}$ remains a delicate step to be considered. In two other publications $[56,57]$, the collected fractions from LLC dimension were just filtrated and injected in LC dimension. In this case, the mobile phases of both dimensions have to be compatible, either close in composition or generating a focusing effect on the head of the LC column. Indeed, the risk of a direct transfer is that the injection on the secondary LC column of a large volume with high elution strength generates dispersion so that the effective number of plates of the secondary LC column is drastically reduced, impairing the resolution power.

For the remaining publications involving LLC and LC in a 2D strategy [58-60], no method for fractions transfer was mentioned.

In order to evaluate the orthogonality of the system sets, the 2D representation of the two dimensional separations were investigated by plotting the Kd values of the compounds from the ${ }^{1} \mathrm{D}$ LLC against the $k$ values of the compounds from the ${ }^{2} D$ LC. The $2 D$ representations are presented in Figure 10 for publications providing adequate retention data $[49,53,57,61]$.

In Zhu et al. in 2009 [49], $n$-hexane/ethyl acetate/methanol/water was used as ${ }^{1} D$ solvent system, the recovered fractions were evaporated to dryness and solubilized in methanol to be transferred to a C18 stationary phase with methanol/water mobile phase. With the elimination of the ${ }^{1} \mathrm{D}$ mobile phase before LC injection, two different dimensions were chosen without any solvent compatibility problems 
and thus two independent separation mechanisms were carried out, as proven by the low correlation in 2D plot (Figure 10a).

In 2015, Zhu et al. implemented a second 2D LLC-HPLC separation [53] with the same transfer strategy. The $2 \mathrm{D}$ representation is shown in Figure $10 \mathrm{~b}$. With the elimination of the ${ }^{1} \mathrm{D}$ mobile phase before $\mathrm{LC}$ injection, the solvent compatibility problems cannot arise. The solvent system $n$-hexane/ethyl acetate/methanol/water was used as ${ }^{1} \mathrm{D}$ solvent system and an ODS stationary phase was implemented with acetonitrile/water mobile phase for the LC separation. Nevertheless, as shown on the figure, the correlation coefficient is close to 1 meaning that the separation mechanisms in the two dimensions are similar for the injected compounds. In this case, orthogonality is really low despite the elimination of the ${ }^{1} \mathrm{D}$ mobile phase before $\mathrm{LC}$ injection was ensured. These two examples show that even when solvent compatibility has been overcome and separation media seem different, the two separation mechanisms need a full investigation to be optimized.

For some examples, no evaporation of the solvents was operated between the two columns and the recovered LLC fractions were directly injected as such in the secondary LC column.

In Wang's work [61], the aqueous-rich ${ }^{1} D$ fraction (composition unknown) is directly sent on ${ }^{2} D$ LC working at $32 \%$ methanol isocratic mobile phase. The first dimension clearly allows the separation of compound 2 with its impurities from compound 1 and compound 4 (with their respective impurities). On the 2D-plot (Figure 10c), it clearly appears that the LLC dimension is used as a basic fractionation step before LC separation whereas the separation mechanisms could be more different and the compounds could be easier to isolate. One can wonder about the usefulness of such a 2D strategy compared to one-dimensional preparative separation.

In Chen's work [57], the ${ }^{1} \mathrm{D}$ mobile phase was essentially water (93\% water, $7 \%$ butanol). Therefore, the mobile phase of the LLC dimension was highly compatible with the reverse phase LC system. The separation mechanisms in the two dimensions are different (quite low correlation coefficient on Figure 10d) even if the two dimensional-separation space is not fully exploited. This case can cause issue for the preparative transfer because of the possible coelution of the peaks.

\section{[here Figure 10]}

In the reported offline LLC-LC papers, the solvent system of the first LLC dimension was chosen with the same criteria than the implementation of unidimensional LLC: suitable Kd values for satisfactory separation time, separation factor higher than 1.5 , satisfactory stationary phase retention and polarity close to the sample polarity if known. Then, the unseparated compounds from the first LLC dimension were injected in HPLC to separate them. No optimization criteria were specifically mentioned for the LC system selection.

With the 1D mobile phase either discarded or compatible, the conditions are fulfilled to offer a wide range of orthogonality sets. Regrettably, with the available data, only one application exhibited satisfactory orthogonality.

\section{2- Sample loop interface}

Compared to the offline mode, the online hyphenation needs a special equipment and technical skills. However, its automation makes it high reproducible and easier to use. The fraction of interest from the ${ }^{1} \mathrm{D}$ LLC has to be transferred in the ${ }^{2} \mathrm{D}$ LC with a special interface such as a sample loop or a solidphase interface.

In 2011 and 2012, Elfakir's group published two applications of two dimensional LLC-HPLC separations for analytical purposes using CPC devices [62,63]. A $20 \mu \mathrm{l}$-sample loop was used with a 6-port switching 
valve as interface between both dimensions. When the valve is in load position, the CPC outlet is sent through the sample loop to the detection system. When the valve is in inject position, the effluent from the CPC column present in the sample loop is sent to HPLC, during that time CPC continues to operate with the CPC effluent sent to the detection system. This interface is the simplest way to implement an online two dimensional LLC-HPLC separation in an instrumental view since the LLC and HPLC techniques have different backpressure and flow rate values. The LLC technique is a low-pressure chromatography (around 70 bars), whereas the HPLC is a high-pressure chromatography (around 400 bars). It is hence preferable to avoid direct injection. The techniques also use different flow rates. Small scale CPC can be employed up to $15 \mathrm{ml} / \mathrm{min}$, whereas analytical HPLC is generally set at around 1 $\mathrm{ml} / \mathrm{min}$. As the switching valve allows a physical separation between the two systems, physical constraints are not a problem anymore.

However, by using a simple sample loop as interface, the mobile phase of the first dimension is still present in the fraction which is transferred to the second dimension. Thus, the compatibility of the mobile phases of both dimensions has to be studied to implement a satisfactory separation. In the cited references, the mobile phase of the first dimension was composed of ethyl acetate/methanol/water with traces of $n$-heptane whereas the mobile phase of the second dimension was acetonitrile/water. The injection solvent is miscible in the ${ }^{2} \mathrm{D}$ mobile phase, but the very high elution strength should result in a very large contribution of injection process on the ${ }^{2} \mathrm{D}$ band broadening. Different parameters were investigated to avoid peak deformation in the secondary separation. With a standard mixture, the injection solvent, the injected volume and the injected amount were studied [62]. They concluded that, according to the HPLC column dispersion, any fraction coming from the CPC column could be injected if the transferred volume did not exceed $20 \mu \mathrm{l}$ ( $2 \% \mathrm{LC}$ column volume). This instrumentation is hence perfectly adapted for analytical purposes or for HPLCguided fractionation. It would be interesting to further develop this approach with a larger preparative LC column as second dimension for preparative purposes.

In 2013, Wei et al. also used this interface with an $8 \mathrm{ml}$-sample loop for the hyphenation of CCC and LC devices at semi-preparative scale [64]. In their work, the strategy to avoid the phase compatibility problems was to use similar mobile phases for the two dimensions. The ${ }^{1} \mathrm{D}$ mobile phase was made of ethyl acetate/methanol/acetonitrile/water (exact composition undefined, but about $30 \%$ water) and the ${ }^{2} \mathrm{D}$ mobile phase was methanol/water $65: 35$. The transferred volume was equivalent to $15 \%$ of the ${ }^{2} \mathrm{D}$ column volume. The risk of this strategy can be the lack of selectivity between the two dimensions due to the use of the same solvents. Unfortunately, retention data were not available in the publication to discuss this point.

\section{3- Solid-phase trapping columns interface}

Qiu's group is the only group to have carried out solid-phase trapping columns as interface for online two dimensional LLC-LC separations. This interface allows the complete elimination of the ${ }^{1} \mathrm{D}$ mobile phase, the focusing of the solutes on the trapping columns and the transfer to HPLC with compatible injection solvent. Four publications have been published in 2014 and 2015 using CCC devices and preparative LC [65-68].

The interface of the first application of Qiu's group in 2014 [65] is presented in Figure 11. The fractions of interest underwent a mixing step in a dynamic mixer to decrease the solvent strength by adding water with a make-up pump. Then, the effluent was sent to a 2-position 10-port valve containing two C18 holding columns allowing the transfer of fractions of interest to second LC dimension. Two trapping columns are implemented in order to allow desorption of the solutes of the LLC fraction trapped on the first trapping column towards LC column while the second LLC fraction is sent to the second trapping column. With this strategy, the entire ${ }^{1} D$ fractions can be sent to the interface for $L C$ injection without stopping the LLC elution. 
By using this interface, the ${ }^{1} \mathrm{D}$ solvents were eliminated. Moreover, with the use of two holding columns, this interface allowed a comprehensive separation in the sense that the entire sample can undergo the two dimensional separation. For this comprehensive mode, the time compatibility issue between both techniques was studied. In the cited reference, the entire LLC peaks were transferred to $L C$ dimension meaning that every LLC peak requires one $L C$ separation run. At preparative scale, the c time separation reached 60 minutes. Thus, to ensure time compatibility, a flow-programming scheme was considered for the LLC implementation. In the flow-programming mode, the LLC dimension was operated at a controlled flow rate to ensure that each LLC fraction eluted within LC separation time frame. The rotation speed was kept the same during the whole LLC separation. This strategy was also applied in 2015 with an instrumental improvement on the water addition before trapping, through the use of a fragmentary dilution and turbulent mixing interface [68].

In 2014 and 2015, Qiu's group illustrated their development through two new 2D LLC-HPLC separation examples $[66,67]$. For these applications, the same instrumentation was enforced with the same two trapping columns. In order to ensure the time compatibility between the two different techniques, a stop-and-go mode was considered for the LLC implementation. While in flow-programming mode, the ${ }^{1} \mathrm{D}$ flow rate was usually slowed down, in the stop-and-go mode, the ${ }^{1} \mathrm{D}$ flow rate was punctually stopped (but not the CCC rotation). Indeed, the processing of the transferred fraction by LC requires around $30 \mathrm{~min}$, which is much more than the time required to fill up a trapping column. Thus while the two trapping columns were processed, the flow rate was stopped in the CCC. It is considered that the band broadening that resulted from diffusion during this holding time was not prejudicial to the ${ }^{1} \mathrm{D}$ separation.

\section{[here Figure 12]}

While developing a 2D method for the isolation of coumarin derivatives from traditional Chinese plant on LLC to avoid irreversible adsorption on silica [66], Qiu's group observed that for some compounds which had close Kd values in LLC, their elution properties, and as first, their selectivity were different in HPLC. After careful investigation concerning these differences, they choose the biphasic LLC solvent system that provided a selectivity as opposite as possible to the LC selectivity. This lead to a very nice multi-targeted separation of 16 compounds (Figure 12) and the isolation for the first time of two minor linear coumarins and one angular coumarin. Here a real two dimensional strategy was carried out in terms of solvent selection and this is the first publication to discuss resolution, efficiency and orthogonality for a two dimensional separation involving LLC two dimensional. For the first time, LLC solvent system was chosen in regards to the complementary selectivity LC provided as well. This resulted in a full usage of the separation space and a globally low correlation coefficient. Looking closer it is however visible that some compounds undergo similar separation mechanisms in the two dimensions (7,8 and 9 in one group, 10,12,13 and 14 in another). Compounds 1 to 5 are separated only thanks to the second dimension. This may cause issue at preparative scale when peaks may coelute due to large loading. On the 2D plot, it is also easy to observe that neither LLC nor LC would have been able to individually perform, as for example compounds 10,12,13,14 coelute in LLC and 3, 8 and 13 would not have been resolved in LC. This success clearly highlights the power of $2 \mathrm{D}$ separations.

While the common sense would consider set the high loading capacity, low efficiency, high selectivity LLC technique before the high resolution LC, it is worth mentioning that some researchers experimented the other way around. In 2013, Shi et al. presented a combination of preparative HPLC and LLC to separate lignane derivatives [69]. They considered that for their application, neither 
preparative HPLC nor LLC could purify all the target active compounds with high purity in one-step separation. Thus, they found that the combination of preparative HPLC and LLC was efficient to reach their goal because of their complementary and orthogonal properties. Surprisingly, in their strategy, HPLC allowed the simplification of complex samples into fractions. They considered that HPLC is a powerful technology with high efficiency and selectivity but that the resolution in preparative LC is fairly low. Effectively, this is the case in their application and on the chromatogram, the resolution was really poor and the selectivity between peaks of interest was clearly low. Only two fractions could be recovered for seven compounds. This is the claimed reason why they used preparative LC as first dimension. Then the LLC separation gave a better selectivity for the peaks and allowed the isolation of the compounds with a high purity. The sample was injected through a preparative LC with a $500 \times 80$ $\mathrm{mm}$ column packed with $5 \mu \mathrm{m}$ octadecylsilane stationary phase. The mobile phase was a gradient composed of ethanol/water. The collected fractions were evaporated to dryness. Only two lyophilized fractions were then sent to LLC separation with two different solvent systems: ethyl acetate-ethanolwater (2:1:3) solvent system and petroleum ether-ethyl acetate-methanol-water (2:5:2:5) solvent system were investigated. This strategy is interesting to investigate nevertheless it remains an unusual one. As said previously, LC is a technique with lower loading capacity than LLC. Thus, it could be better to enforce LLC as first dimension to make use of this advantage and to benefit from more chromatographic plates in the second dimension to achieve proper resolution.

\section{Conclusion and outlook}

The two dimensional separations combining LLC technique with LLC or HPLC techniques are interesting at preparative scale in order to isolate and purify compounds from a highly complex sample. From all the investigated publications, including LLC-LLC and LLC-HPLC hyphenations, $90 \%$ of the applications are implemented at preparative scale for further NMR identification of isolated compounds and so for the production of high quality standards.

The two dimensional separations involve different separation mechanisms meaning different selectivities and high orthogonality between the two dimensions. In order to get these different separation mechanisms, a solvent switch should be performed between first and second dimension to generate new column-solutes interactions. Thus, solvents compatibility problems can occur between the dimensions. These issues are more significant when the second dimension possesses a liquid stationary phase as the physical stability issue adds to the band broadening issue. The type of interface between the dimensions has hence a major influence on the success of hyphenation. If there is no compatibility problems between the two dimensions solvents (or if they are minimized by small fraction volumes injection), the injection of the ${ }^{1} D$ fraction to the second dimension can be achieved in online mode by a direct transfer using a switching valve or a sample loop to control the injection volume. To avoid any compatibility issue, the removal of the ${ }^{1} \mathrm{D}$ mobile phase solvents can be achieved either off-line or online mode using solid-phase trapping columns.

The instrumental evolution shall allow an improvement of the orthogonality due to the possible selectivity difference between both dimensions. Regrettably, this was not often the case in the examples. A slight change in solvent system composition was mostly observed and no global two dimensional strategy was enforced to select the solvent systems which could give the best orthogonality. The LLC-LLC hyphenation was mainly used as a gradient strategy with a slight change in solvent system composition to separate closely related compounds.

Beyond the instrumental evolution, the priority to enforce a two dimensional separation using the LLC technique with a high peak capacity (either with another LLC or with LC) must be set on a global two dimensional methodology to select the chromatographic columns offering the best orthogonality. Considering 2D-plot at the first stage of method development is one of the paths that can be taken for 
that purpose. Then multiple-targeted compounds and / or comprehensive purifications could offer 671 more to the discovery of new molecules.

672 
[1] Y. Ito, Origin and Evolution of the Coil Planet Centrifuge: A Personal Reflection of My 40 Years of CCC Research and Development, Separation \& Purification Reviews, 34 (2005) 131-154.

678 [2] L.C. Craig, O. Post, Apparatus for Countercurrent Distribution, Analytical Chemistry, 21 (1949) 500679504.

680 [3] W.D. Conway, Counter-current chromatography: Simple process and confusing terminology,

[4] S.M.P. Guido F Pauli, J Brent Friesen, Countercurrent Separation of Natural Products, J. Nat. Prod., 71 (2008) 1489-1508.

[5] Y. Ito, J.-M. Menet, Coil planet centrifuges for High-Speed countercurrent chromatography, in: J.M. Menet, D. Thiébaut (Eds.) Countercurrent Chromatography, Marcel Dekker, 1999, pp. 87-119.

[6] A. Berthod, T. Maryutina, B. Spivakov, O. Shpigun, I.A. Sutherland, Countercurrent chromatography in analytical chemistry: (IUPAC Technical Report), Pure and Applied Chemistry, (2009) 355.

[7] A. Berthod, K. Talabardon, Centrifugal Partition Chromatography: operating parameters and partition coefficient determination, in: J.-M. Menet, D. Thiébaut (Eds.) Countercurrent chromatography, Marcel Dekker, 1999, pp. 121-148.

[8] W.D. Conway, Countercurrent Chromatography: Apparatus, Theory and Applications, VCH Publishers, 1990.

[9] A. Berthod, Countercurrent Chromatography the support-free liquid stationary phase, in: Comprehensive analytical chemistry, Elsevier Science, 2002.

[10] K. Skalicka-Wozniak, I. Garrard, A comprehensive classification of solvent systems used for natural product purifications in countercurrent and centrifugal partition chromatograhy, Natural Product Reports, (2015) 1556-1561.

[11] A. Berthod, M.J. Ruiz-Ángel, S. Carda-Broch, Countercurrent chromatography: People and applications, Journal of Chromatography A, 1216 (2009) 4206-4217.

[12] R. Hu, Y. Pan, Recent trends in counter-current chromatography, TrAC Trends in Analytical Chemistry, 40 (2012) 15-27.

[13] J.B.M. J Brent Friesen, Shao-Nong Chen, Guido F Pauli, Countercurrent separation of natural products: an update, journal of natural products, 78 (2015) 1765-1796.

[14] L. Yin, Y. Li, B. Lu, Y. Jia, J. Peng, Trends in Counter-Current Chromatography: Applications to Natural Products Purification, Separation \& Purification Reviews, 39 (2010) 33-62.

[15] T. Michel, E. Destandau, C. Elfakir, New advances in countercurrent chromatography and centrifugal partition chromatography: focus on coupling strategy, Analytical \& Bioanalytical Chemistry, 406 (2014) 957-969.

[16] X.-M. Xie, W.-Y. Sun, J.-Y. Huang, N. Polachi, L. Tong, G.-X. Sun, Preparative High Performance Liquid Chromatography-based Multidimensional Chromatography and Its Application in Traditional Chinese Medicine, Chinese Journal of Analytical Chemistry, 44 (2016) 1140-1147.

[17] T. Kapp, W. Vetter, Offline coupling of high-speed counter-current chromatography and gas chromatography/mass spectrometry generates a two-dimensional plot of toxaphene components, Journal of Chromatography A, 1216 (2009) 8391-8397.

[18] T. Inui, Y. Wang, S.M. Pro, S.G. Franzblau, G.F. Pauli, Unbiased evaluation of bioactive secondary metabolites in complex matrices, Fitoterapia, 83 (2012) 1218-1225.

[19] D.R. Stoll, X. Li, X. Wang, P.W. Carr, S.E.G. Porter, S.C. Rutan, Fast, comprehensive two-dimensional liquid chromatography, Journal of Chromatography A, 1168 (2007) 3-43.

[20] P. Dugo, F. Cacciola, T. Kumm, G. Dugo, L. Mondello, Comprehensive multidimensional liquid chromatography: Theory and applications, Journal of Chromatography A, 1184 (2008) 353-368.

[21] G. Guiochon, N. Marchetti, K. Mriziq, R.A. Shalliker, Implementations of two-dimensional liquid chromatography, Journal of Chromatography A, 1189 (2008) 109-168.

[22] I. François, K. Sandra, P. Sandra, Comprehensive liquid chromatography: Fundamental aspects and practical considerations-A review, Analytica Chimica Acta, 641 (2009) 14-31. 
[23] P.J. Marriott, Z.-y. Wu, P. Schoenmakers, Nomenclature and conventions in comprehensive multidimensional chromatography - An update, LCGC Europe, 25 (2012).

[24] M. Gilar, P. Olivova, A.E. Daly, J.C. Gebler, Orthogonality of Separation in Two-Dimensional Liquid Chromatography, Analytical Chemistry, 77 (2005) 6426-6434.

[25] M. Gilar, J. Fridrich, M.R. Schure, A. Jaworski, Comparison of Orthogonality Estimation Methods for the Two-Dimensional Separations of Peptides, Analytical Chemistry, 84 (2012) 8722-8732.

[26] P.J. Slonecker, X. Li, T.H. Ridgway, J.G. Dorsey, Informational Orthogonality of Two-Dimensional Chromatographic Separations, Analytical Chemistry, 68 (1996) 682-689.

[27] L.I.U. Zaiyou, D.G.J. Patterson, M.L. Lee, Geometric approach to factor analysis for the estimation of orthogonality and practical peak capacity in comprehensive two-dimensional separations (English), Analytical chemistry (Washington, DC), 67 (1995) 3840-3845.

[28] S.C. Rutan, J.M. Davis, P.W. Carr, Fractional coverage metrics based on ecological home range for calculation of the effective peak capacity in comprehensive two-dimensional separations, Journal of Chromatography A, 1255 (2012) 267-276.

[29] W. Nowik, S. Héron, M. Bonose, M. Nowik, A. Tchapla, Assessment of Two-Dimensional Separative Systems Using Nearest-Neighbor Distances Approach. Part 1: Orthogonality Aspects, Analytical Chemistry, 85 (2013) 9449-9458.

[30] W. Nowik, M. Bonose, S. Héron, M. Nowik, A. Tchapla, Assessment of Two-Dimensional Separative Systems Using the Nearest Neighbor Distances Approach. Part 2: Separation Quality Aspects, Analytical Chemistry, 85 (2013) 9459-9468.

[31] M. Camenzuli, P.J. Schoenmakers, A new measure of orthogonality for multi-dimensional chromatography, Analytica Chimica Acta, 838 (2014) 93-101.

[32] F. Yang, J. Quan, T.Y. Zhang, Y. Ito, Multidimensional counter-current chromatographic system and its application, Journal of Chromatography A, 803 (1998) 298-301.

[33] G. Tian, T. Zhang, Y. Zhang, Y. Ito, Separation of tanshinones from Salvia miltiorrhiza Bunge by multidimensional counter-current chromatography, Journal of Chromatography A, 945 (2002) 281285.

[34] J. Wei, Z. Tianyou, I. Yoichiro, Preparative Separation of Tripdiolide from Chinese Traditional Herb by Multidimensional CCC, Journal of Liquid Chromatography \& Related Technologies, 28 (2005) 19031911.

[35] Y. Wei, Y. Ito, Preparative isolation of imperatorin, oxypeucedanin and isoimperatorin from traditional Chinese herb "bai zhi" Angelica dahurica (Fisch. ex Hoffm) Benth. et Hook using multidimensional high-speed counter-current chromatography, Journal of Chromatography A, 1115 (2006) 112-117.

[36] M. Englert, L. Brown, W. Vetter, Heart-Cut Two-Dimensional Countercurrent Chromatography with a Single Instrument, Analytical Chemistry, 87 (2015) 10172-10177.

[37] Y. Lu, C. Sun, R. Liu, Y. Pan, Effective two-dimensional counter-current chromatographic method for simultaneous isolation and purification of oridonin and ponicidin from the crude extract of Rabdosia rubescens, Journal of Chromatography A, 1146 (2007) 125-130.

[38] Y. Lu, R. Hu, Z. Dai, Y. Pan, Preparative separation of anti-oxidative constituents from Rubia cordifolia by column-switching counter-current chromatography, Journal of Separation Science, 33 (2010) 2200-2205.

[39] Y. Lu, C. Sun, Y. Wang, Y. Pan, Two-dimensional counter-current chromatography for the preparative separation of prenylflavonoids from Artocarpus altilis, Journal of Chromatography A, 1151 (2007) 31-36.

[40] R.H. Yanbin Lu, Yuanjiang Pan, Integrated Countercurrent Extraction of Natural Products: A Combination of Liquid and Solid Supports, Analytical Chemistry, 82 (2010) 3081-3085.

[41] R. Hu, X. Dai, X. Xu, C. Sun, Y. Pan, Two-dimensional counter-current chromatography: 1st Traditional counter-current chromatography, 2nd acid-base elution counter-current chromatography, Journal of Chromatography A, 1218 (2011) 6085-6091.

[42] Q. Liu, S. Shi, L. Liu, H. Yang, W. Su, X. Chen, Separation and purification of bovine serum albumin binders from Fructus polygoni orientalis using off-line two-dimensional complexation high-speed 
counter-current chromatography target-guided by ligand fishing, Journal of Chromatography A, 1304 (2013) 183-193.

[43] K. Faure, E. Bouju, P. Suchet, A. Berthod, Use of Limonene in Countercurrent Chromatography: A Green Alkane Substitute, Analytical chemistry, 85 (2013) 4644-4650.

[44] Q. Liu, H. Zeng, S. Jiang, L. Zhang, F. Yang, X. Chen, H. Yang, Separation of polyphenols from leaves of Malus hupehensis (Pamp.) Rehder by off-line two-dimensional High Speed Counter-Current Chromatography combined with recycling elution mode, Food Chemistry, 186 (2015) 139-145.

[45] S. Jiang, Q. Liu, Y. Xie, H. Zeng, L. Zhang, X. Jiang, X. Chen, Separation of five flavonoids from tartary buckwheat (Fagopyrum tataricum (L.) Gaertn) grains via off-line two dimensional high-speed countercurrent chromatography, Food Chemistry, 186 (2015) 153-159.

[46] H. Zeng, Q. Liu, M. Wang, S. Jiang, L. Zhang, X. He, J. Wang, X. Chen, Target-guided separation of antioxidants from Semen cassia via off-line two-dimensional high-speed counter-current chromatography combined with complexation and extrusion elution mode, Journal of Chromatography B, 1001 (2015) 58-65.

[47] F.d.N. Costa, G. Jerz, F.d.S. Figueiredo, P. Winterhalter, G.G. Leitão, Solvent system selectivities in countercurrent chromatography using Salicornia gaudichaudiana metabolites as practical example with off-line electrospray mass-spectrometry injection profiling, Journal of Chromatography A, 1385 (2015) 20-27.

[48] J.C.T. Silva, G.N. Jham, R.D.a.L. Oliveira, L. Brown, Purification of the seven tetranortriterpenoids in neem (Azadirachta indica) seed by counter-current chromatography sequentially followed by isocratic preparative reversed-phase high-performance liquid chromatography, Journal of Chromatography A, 1151 (2007) 203-210.

[49] L. Zhu, H. Li, Y. Liang, X. Wang, H. Xie, T. Zhang, Y. Ito, Application of high-speed counter-current chromatography and preparative high-performance liquid chromatography mode for rapid isolation of anthraquinones from Morinda officinalis How, Separation and Purification Technology, 70 (2009) 147152.

[50] Z. Hou, J. Luo, J. Wang, L. Kong, Separation of minor coumarins from Peucedanum praeruptorum using HSCCC and preparative HPLC guided by HPLC/MS, Separation and Purification Technology, 75 (2010) 132-137.

[51] T. Chen, Y. Liu, D. Zou, C. Chen, J. You, G. Zhou, J. Sun, Y. Li, Application of an efficient strategy based on liquid-liquid extraction, high-speed counter-current chromatography, and preparative HPLC for the rapid enrichment, separation, and purification of four anthraquinones from Rheum tanguticum, Journal of Separation Science, 37 (2014) 165-170.

[52] H. Ying, J. Liu, Q. Du, Analysis and determination of oestrogen-active compounds in fructus amomi by the combination of high-speed counter-current chromatography and high performance liquid chromatography, Journal of Chromatography B, 958 (2014) 36-42.

[53] L. Zhu, B. Li, X. Liu, G. Huang, X. Meng, Purification of six lignans from the stems of Schisandra chinensis by using high-speed counter-current chromatography combined with preparative highperformance liquid chromatography, Food Chemistry, 186 (2015) 146-152.

[54] W. Guo, L. Wang, Y. Gao, B. Zhao, D. Wang, W. Duan, Z. Yu, Isolation of isochlorogenic acid isomers in flower buds of Lonicera japonica by high-speed counter-current chromatography and preparative high performance liquid chromatography, Journal of Chromatography B, 981-982 (2015) 27-32.

[55] T. Chen, H. Li, D. Zou, Y. Liu, C. Chen, G. Zhou, Y. Li, Separation of three anthraquinone glycosides including two isomers by preparative high-performance liquid chromatography and high-speed countercurrent chromatography from Rheum tanguticum Maxim ex. Balf, Journal of Separation Science, 39 (2016) 3105-3112.

[56] D. de Beer, G. Jerz, E. Joubert, V. Wray, P. Winterhalter, Isolation of isomangiferin from honeybush (Cyclopia subternata) using high-speed counter-current chromatography and high-performance liquid chromatography, Journal of Chromatography A, 1216 (2009) 4282-4289.

[57] D. Chen, J. Fan, P. Wang, L. Zhu, Y. Jin, Y. Peng, S. Du, Isolation, identification and antioxidative capacity of water-soluble phenylpropanoid compounds from Rhodiola crenulata, Food Chemistry, 134 (2012) 2126-2133. 

preparative high-performance liquid chromatography to separate galactolipids from pumpkin, Journal of Chromatography A, 1216 (2009) 4176-4180. [59] Q.-B. Han, Y. Zhou, C. Feng, G. Xu, S.-X. Huang, S.-L. Li, C.-F. Qiao, J.-Z. Song, D.C. Chang, K.Q. Luo, H.-X. Xu, Bioassay guided discovery of apoptosis inducers from gamboge by high-speed countercurrent chromatography and high-pressure liquid chromatography/electrospray ionization quadrupole time-of-flight mass spectrometry, Journal of Chromatography B, 877 (2009) 401-407.

[60] G.-j.-s. Cheng, G.-k. Li, X.-h. Xiao, Microwave-assisted extraction coupled with counter-current chromatography and preparative liquid chromatography for the preparation of six furocoumarins from Angelica Pubescentis Radix, Separation and Purification Technology, 141 (2015) 143-149.

[61] X. Wang, Y. Liang, L. Zhu, H. Xie, H. Li, J. He, M. Pan, T. Zhang, Y. Ito, Preparative isolation and purification of flavone $\mathrm{C}$-glycosides from the leaves of ficus microcarpa by medium-pressure liquid chromatography, high speed countercurrent chromatography and preparative liquid chromatography, Journal of Liquid Chromatography \& Related Technologies, 33 (2010) 462-480.

[62] T. Michel, E. Destandau, C. Elfakir, On-line hyphenation of centrifugal partition chromatography and high pressure liquid chromatography for the fractionation of flavonoids from Hippophaë rhamnoides L. berries, Journal of Chromatography A, 1218 (2011) 6173-6178.

[63] T. Michel, E. Destandau, L. Fougère, C. Elfakir, New 'hyphenated' CPC-HPLC-DAD-MS strategy for simultaneous isolation, analysis and identification of phytochemicals: application to xanthones from Garcinia mangostana, Analytical \& Bioanalytical Chemistry, 404 (2012) 2963-2972.

[64] Y. Wei, W. Huang, Y. Gu, Online isolation and purification of four phthalide compounds from Chuanxiong rhizoma using high-speed counter-current chromatography coupled with semipreparative liquid chromatography, Journal of Chromatography A, 1284 (2013) 53-58.

[65] Y.-K. Qiu, X. Yan, M.-J. Fang, L. Chen, Z. Wu, Y.-F. Zhao, Two-dimensional countercurrent chromatography $\times$ high performance liquid chromatography for preparative isolation of toad venom, Journal of Chromatography A, 1331 (2014) 80-89.

[66] J.-L. Liu, X.-Y. Wang, L.-L. Zhang, M.-J. Fang, Y.-L. Wu, Z. Wu, Y.-K. Qiu, Two-dimensional countercurrent chromatography $\times$ high performance liquid chromatography with heart-cutting and stop-and-go techniques for preparative isolation of coumarin derivatives from Peucedanum praeruptorum Dunn, Journal of Chromatography A, 1374 (2014) 156-163.

[67] W.-B. Chen, S.-Q. Li, L.-J. Chen, M.-J. Fang, Q.-C. Chen, Z. Wu, Y.-L. Wu, Y.-K. Qiu, Online polar two phase countercurrent chromatography $x$ high performance liquid chromatography for preparative isolation of polar polyphenols from tea extract in a single step, Journal of Chromatography B-Analytical Technologies in the Biomedical and Life Sciences, 997 (2015) 179-186.

[68] D. Wang, L.-J. Chen, J.-L. Liu, X.-Y. Wang, Y.-L. Wu, M.-J. Fang, Z. Wu, Y.-K. Qiu, On-line twodimensional countercurrent chromatography $\times$ high performance liquid chromatography system with a novel fragmentary dilution and turbulent mixing interface for preparation of coumarins from Cnidium monnieri, Journal of Chromatography A, 1406 (2015) 215-223.

[69] S.-Y. Shi, M.-J. Peng, Y.-P. Zhang, S. Peng, Combination of preparative HPLC and HSCCC methods to separate phosphodiesterase inhibitors from Eucommia ulmoides bark guided by ultrafiltrationbased ligand screening, Analytical \& Bioanalytical Chemistry, 405 (2013) 4213-4223.

[70] Q. Liu, J. Zhou, J. Yu, Y. Xie, X. Jiang, H. Yang, X. Chen, Systematic and efficient separation of 11 compounds from Rhizoma Chuanxiong via counter-current chromatography-solid phase extractioncounter-current chromatography hyphenation, Journal of Chromatography A, 1364 (2014) 204-213. 
Figure 1. Schematic design of (a) Countercurrent chromatography coil and (b) Centrifugal partition 876 chromatography rotor.

877 Figure 2. 2D orthogonality representation of two dimensional separations: (a) strong correlation 878 coefficient $r^{2}$; (b) low correlation coefficient $r^{2}$ and low utilization of the 2D space; (c) low correlation coefficient $r^{2}$ without optimized utilization of the 2D space; (d) low correlation coefficient $r^{2}$ and optimized utilization of the 2D space. Modified from [19].

Figure 3. First online two dimensional LLC-LLC instrumentation using a 6-port switching valve as interface between ${ }^{1} \mathrm{D}$ LLC and ${ }^{2} \mathrm{D}$ LLC [32].

Figure 4. 2D orthogonality representation of a two dimensional LLC-LLC separation using switching valve interface. Data from reference [36]. ${ }^{1} \mathrm{D}$ solvent system: $n$-hexane/methyl tert-butyl ether/methanol/water 5:2:5:3 and ${ }^{2} \mathrm{D}$ solvent system: cyclohexane/methyl tert-butyl ether/methanol/water 5:2:5:3.

Figure 5. Homemade column switching system interface used by Pan's group between ${ }^{1} D$ LLC and ${ }^{2} D$ LLC. Modified from [37].

Figure 6. Solid-phase trapping column interface used by Pan's group between ${ }^{1} \mathrm{D}$ LLC and ${ }^{2} \mathrm{D}$ LLC. Modified from [40].

Figure 7. 2D orthogonality representation of a two dimensional LLC-LLC separation using solid-phase trapping columns interface. Data from reference [41]. ${ }^{1} \mathrm{D}$ solvent system: $n$-hexane/ethyl acetate/methanol/water 1:1:1:1 and ${ }^{2} \mathrm{D}$ solvent system: methyl tert-butyl ether/acetonitrile/water 2:2:3.

Figure 8. 2D orthogonality representations two dimensional LLC-LLC separations in offline mode: (a) ${ }^{1} \mathrm{D}$ solvent system: $\mathrm{n}$-hexane/ethyl acetate/methanol/water 3:5:3:5 and ${ }^{2} \mathrm{D}$ solvent system: ethyl acetate/n-butanol/water 7:3:10, data from [45]; (b) ${ }^{1} \mathrm{D}$ solvent system: petroleum ether/ethyl acetate/methanol/water $1: 1: 1: 1$ and ${ }^{2} \mathrm{D}$ solvent system: petroleum ether/ethyl acetate/methanol/water $+\mathrm{Cu}^{2+} 1: 1: 1: 1$, data from [46].

Figure 9. Experimental 2D-plot implemented in das Neves Costa et al. solvent system selection approach. Modified from [47].

Figure 10. 2D orthogonality representations of offline LLC-HPLC two dimensional separations: (a) ${ }^{1} \mathrm{D}$ solvent system: $n$-hexane/ethyl acetate/methanol/water 6:4:5:5 and ${ }^{2} \mathrm{D}$ system: $\mathrm{C} 18$ in methanol/water 60:40 isocratic mobile phase; Data from Zhu et al. [49]: (b) ${ }^{1} D$ solvent system: $n$ hexane/ethyl acetate/methanol/water 1:1:1:1 and ${ }^{2} D$ system: ODS in acetonitrile/water 50:50 isocratic mobile phase; Data from Zhu et al. [53]: (c) ${ }^{1} D$ solvent system: methyl tert-butyl ether/ethyl acetate/1butanol/acetonitrile/modified water 1:3:1:1:5 and ${ }^{2} D$ system: ODS in methanol/water isocratic mobile 908 phase; Data from Wang et al. [60] and (d) ${ }^{1} D$ solvent system: ethyl acetate/ $n$-butanol/water 0.5:4.5:5 909 and ${ }^{2} \mathrm{D}$ system: $\mathrm{C} 18$ in methanol/water isocratic mobile phase; Data from Chen et al. [57]

Figure 11. Solid-phase trapping columns interface between $1^{\text {st }}$ dimensional LLC and $2^{\text {nd }}$ dimensional HPLC. Modified from [65].

Figure 12. Two dimensional LLC-HPLC separation using solid-phase trapping columns interface and stop-and-go mode [66] and its corresponding $2 \mathrm{D}$ orthogonality representation: ${ }^{1} \mathrm{D}$ solvent system: $n$ heptane/acetone/water 31:50:19 and ${ }^{2} \mathrm{D}$ system: $\mathrm{C} 18$ in methanol/water gradient as mobile phase. 
(a)

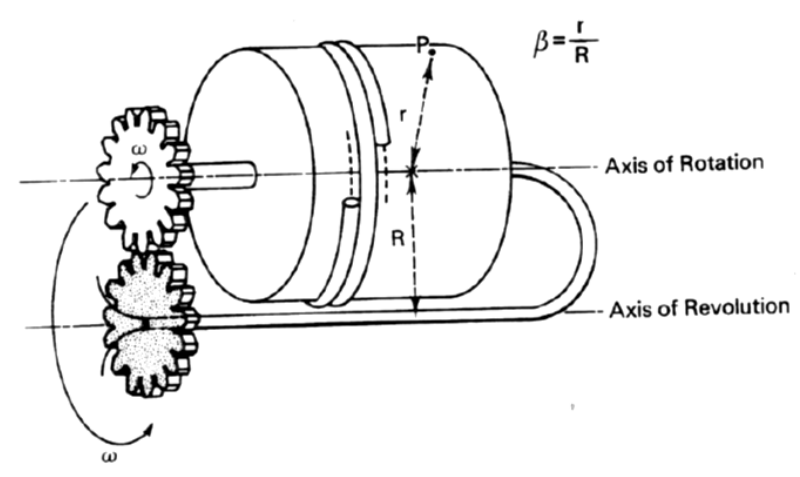

(b)
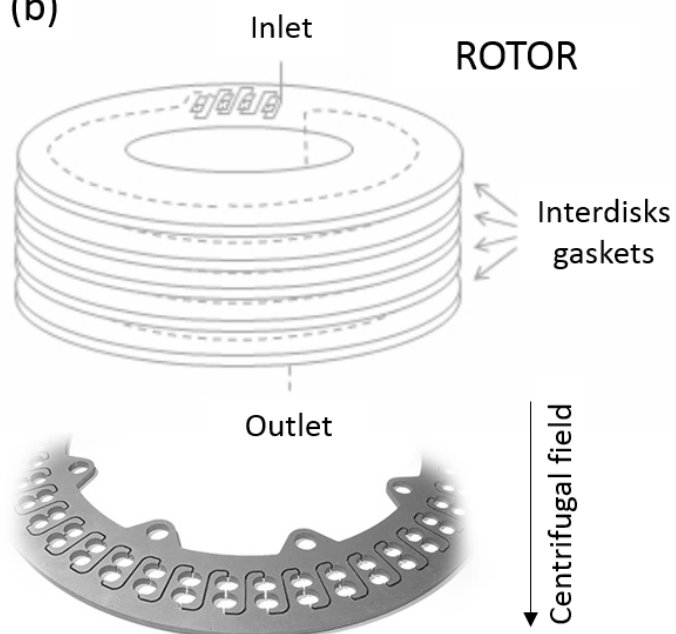

915

916 Figure 1. Schematic design of (a) Countercurrent chromatography coil and (b) Centrifugal partition 917 chromatography rotor. 
(a)

$r^{2}=1$

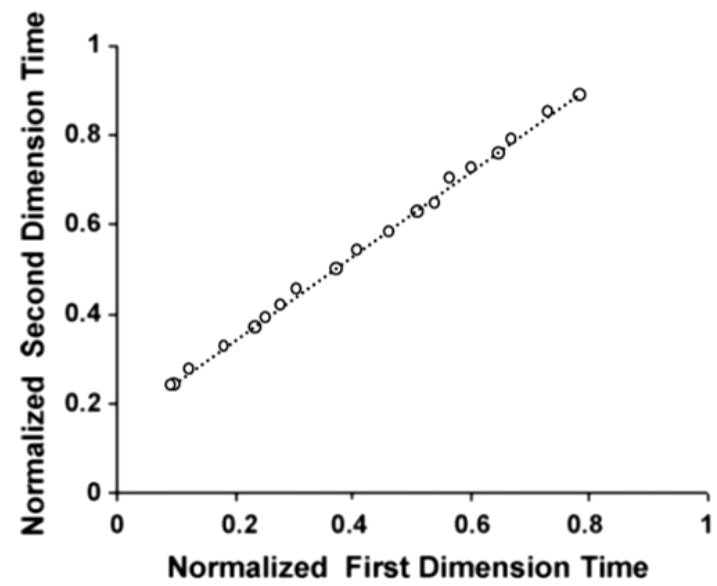

(c)
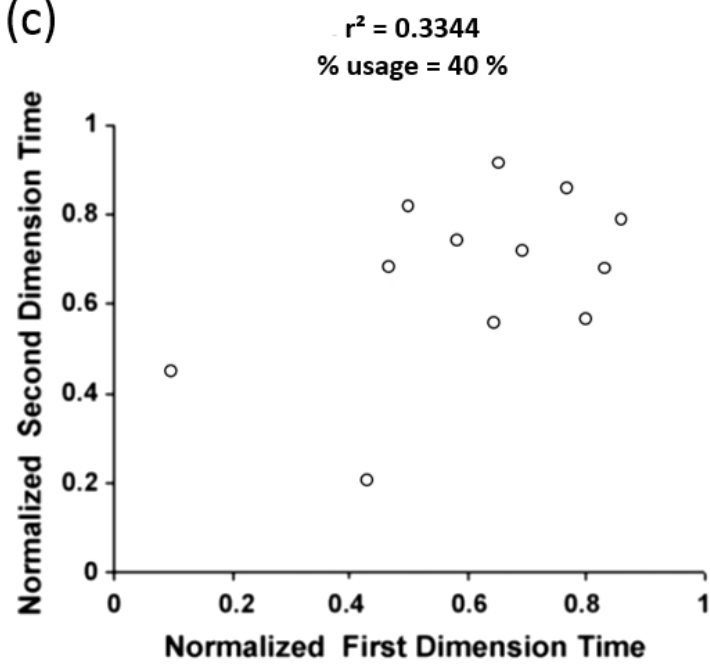

(b)

$r^{2}=0.0005$

$\%$ usage $=10 \%$

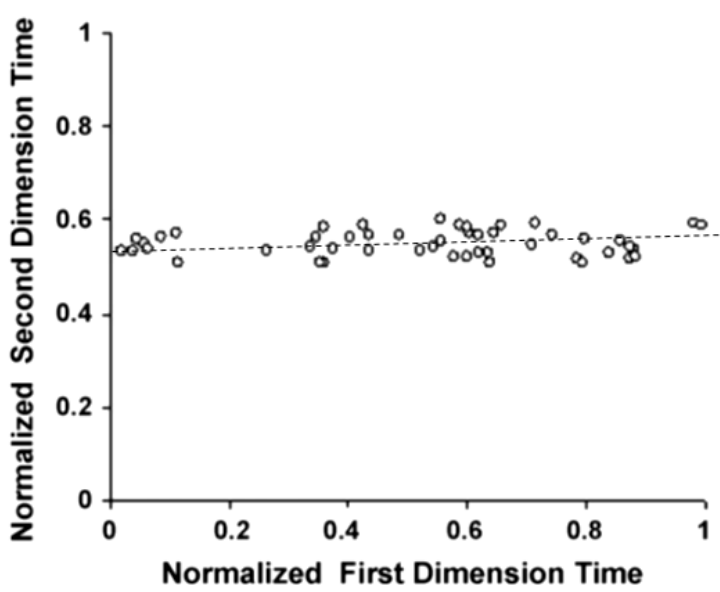

(d)

$$
r^{2}=0.0005
$$

$\%$ usage $=100 \%$

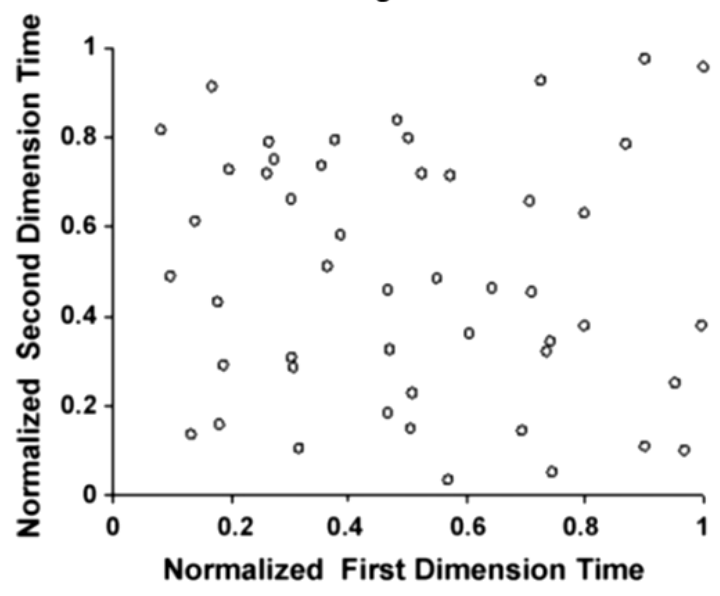

Figure 2. 2D orthogonality representation of two dimensional separations: (a) strong correlation coefficient $r^{2}$; (b) low correlation coefficient $r^{2}$ and low utilization of the 2D space; (c) low correlation coefficient $r^{2}$ without optimized utilization of the 2D space; (d) low correlation coefficient $r^{2}$ and optimized utilization of the 2D space. Modified from [19]. 


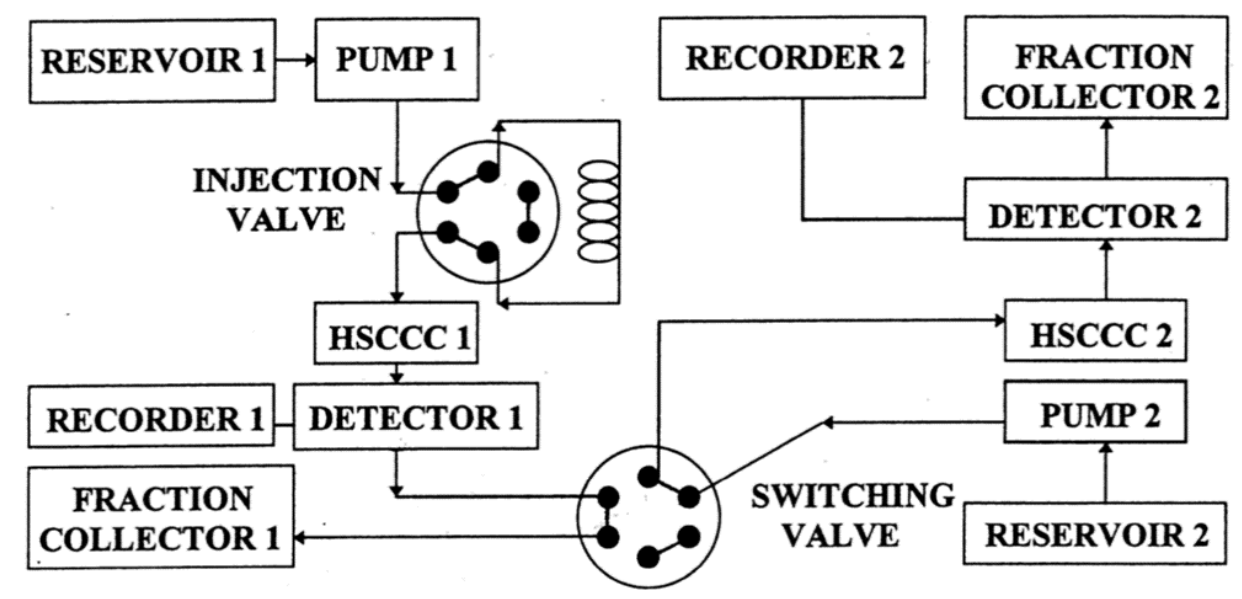

926

Figure 3. First online two dimensional LLC-LLC instrumentation using a 6-port switching valve as interface between ${ }^{1} \mathrm{D}$ LLC and ${ }^{2} \mathrm{D}$ LLC [32].

929

930

931

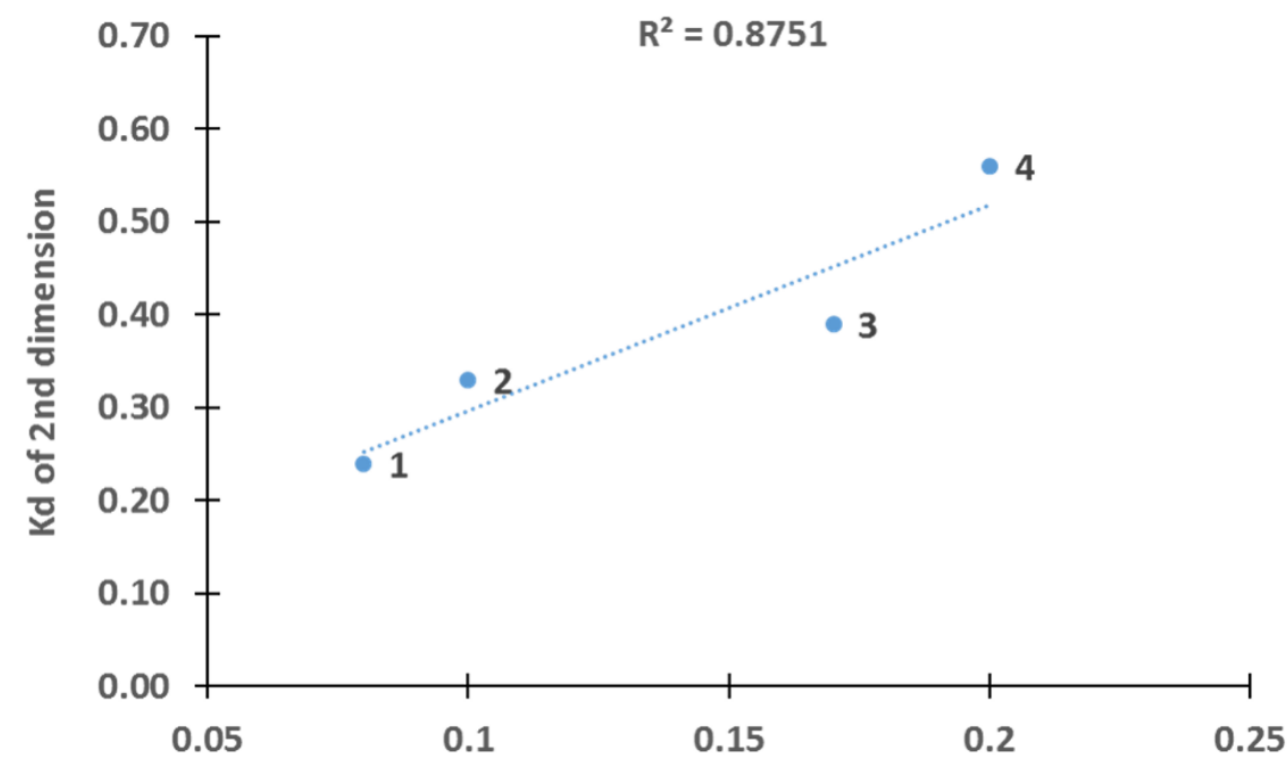

Kd of 1st dimension

933 Figure 4. 2D orthogonality representation of a two dimensional LLC-LLC separation using switching 934 valve interface. Data from reference [36]. ${ }^{1} \mathrm{D}$ solvent system: $n$-hexane/methyl tert-butyl 935 ether/methanol/water 5:2:5:3 and ${ }^{2} \mathrm{D}$ solvent system: cyclohexane/methyl tert-butyl ether/methanol/water 5:2:5:3. 


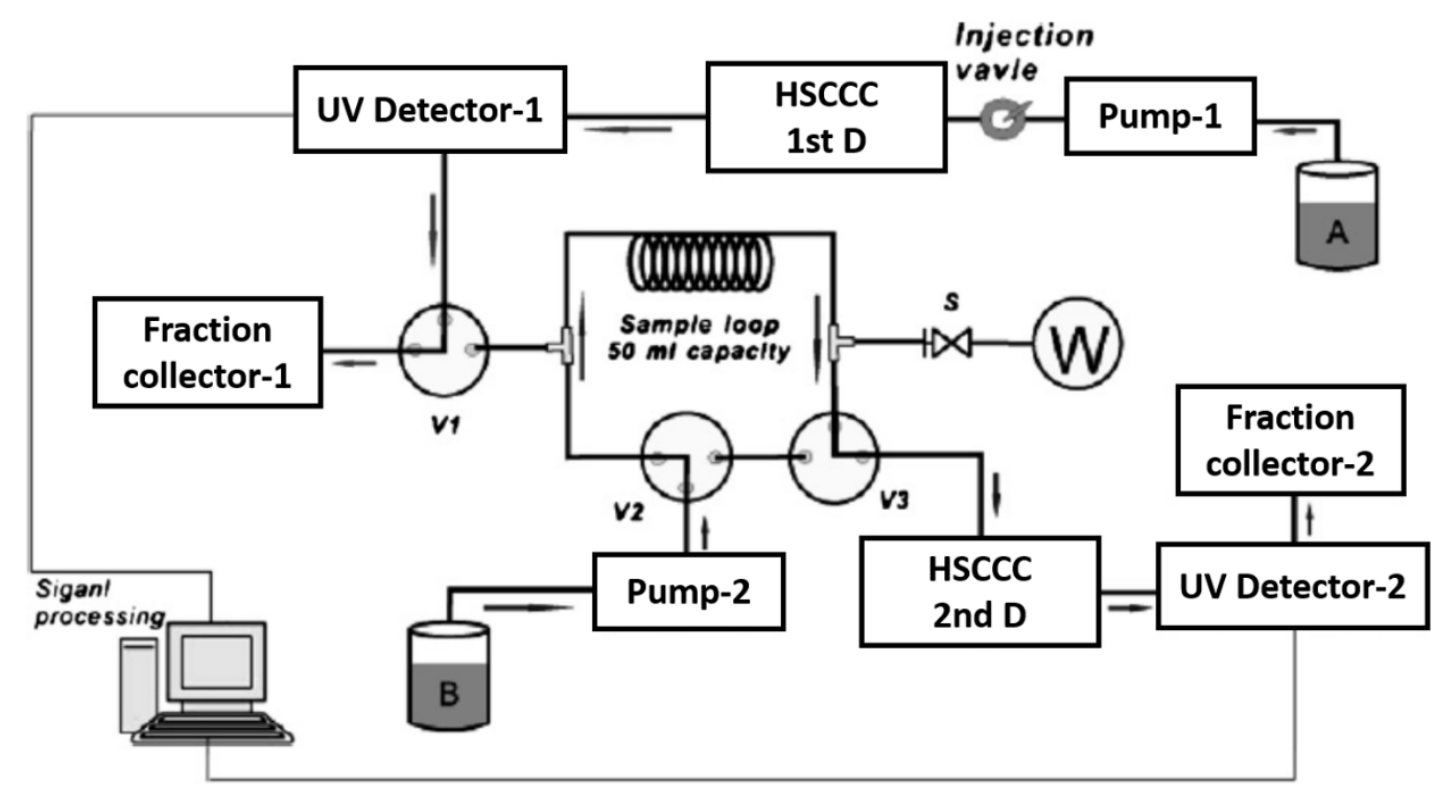

938

939

940

941

942

943

944 Figure 6. Solid-phase trapping column interface used by Pan's group between ${ }^{1} \mathrm{D}$ LLC and ${ }^{2} \mathrm{D}$ LLC. 945 Modified from [40].

Figure 5. Homemade column switching system interface used by Pan's group between ${ }^{1} D$ LLC and ${ }^{2} D$ LLC. Modified from [37].
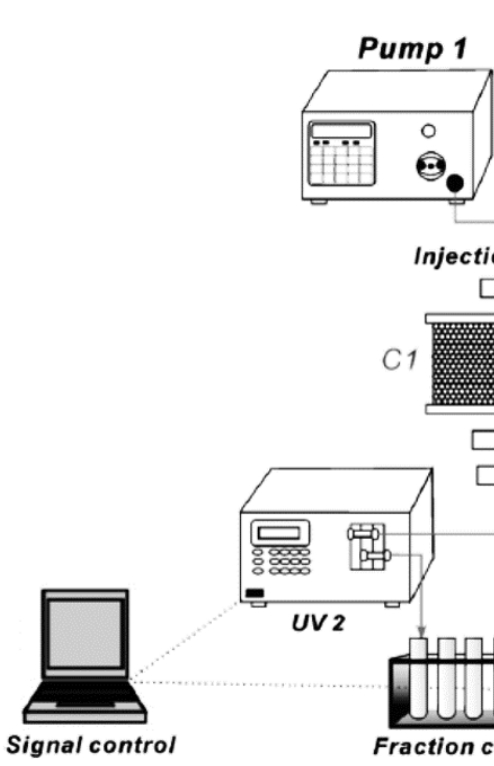

Solid-phase
Trapping Int

Trapping Interface

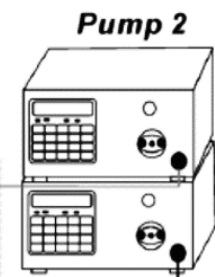

Injection valve

C1

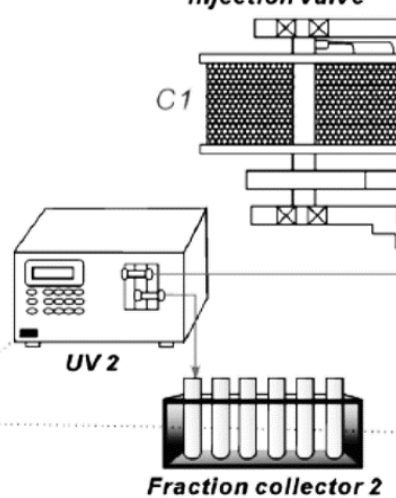

M
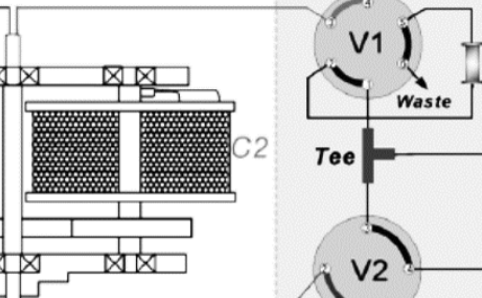

Make-up Make-
Pump

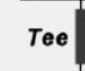

v2

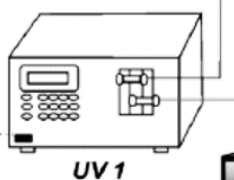

$N_{2}$ 


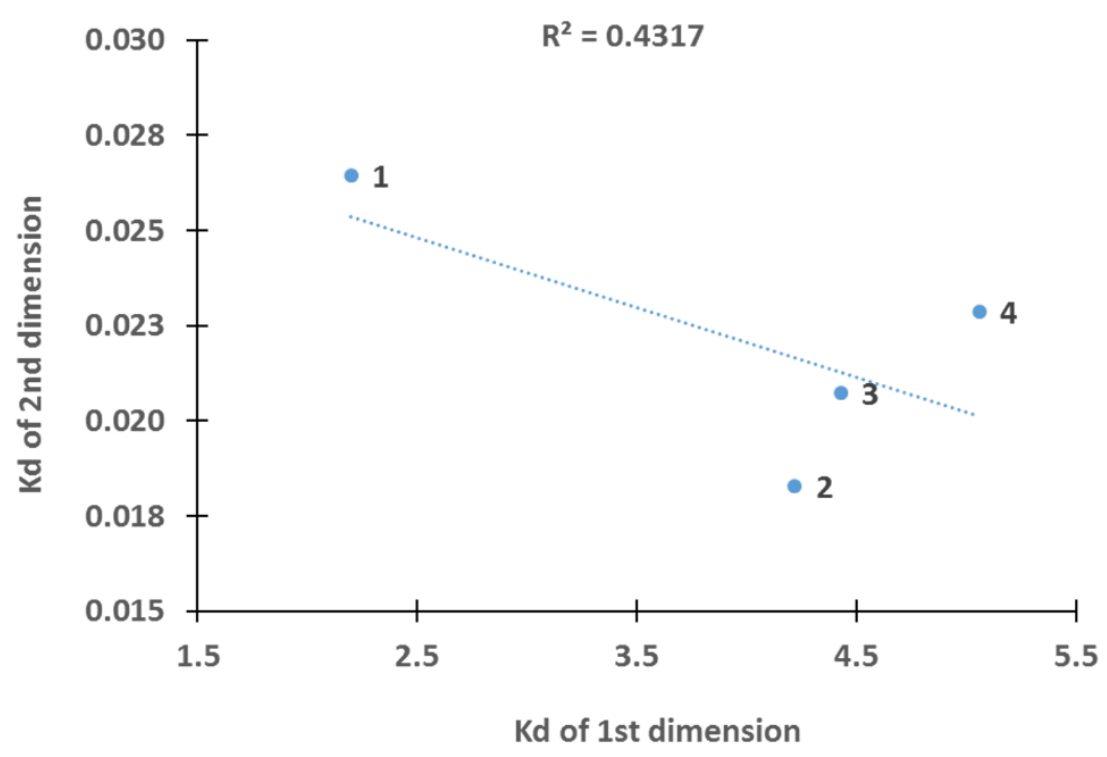

947

Figure 7. 2D orthogonality representation of a two dimensional LLC-LLC separation using solid-phase 949 trapping columns interface. Data from reference [41]. ${ }^{1} \mathrm{D}$ solvent system: $n$-hexane/ethyl 950 acetate/methanol/water 1:1:1:1 and ${ }^{2} \mathrm{D}$ solvent system: methyl tert-butyl ether/acetonitrile/water $951 \quad 2: 2: 3$.

952

953

(a)

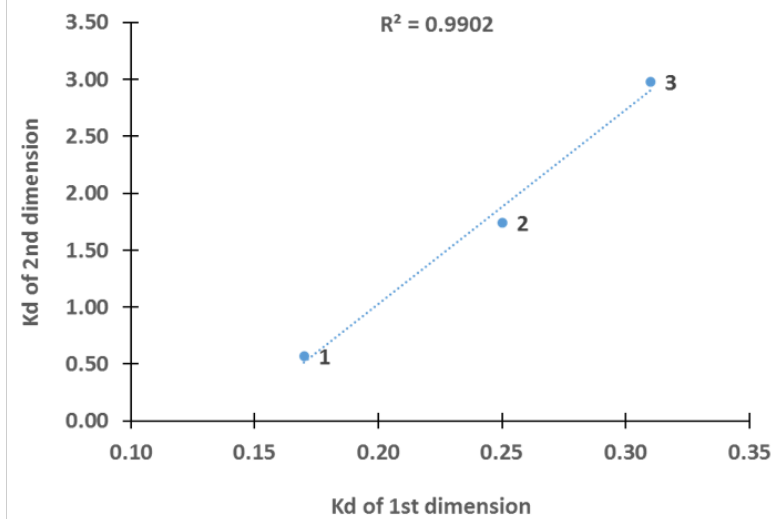

(b)

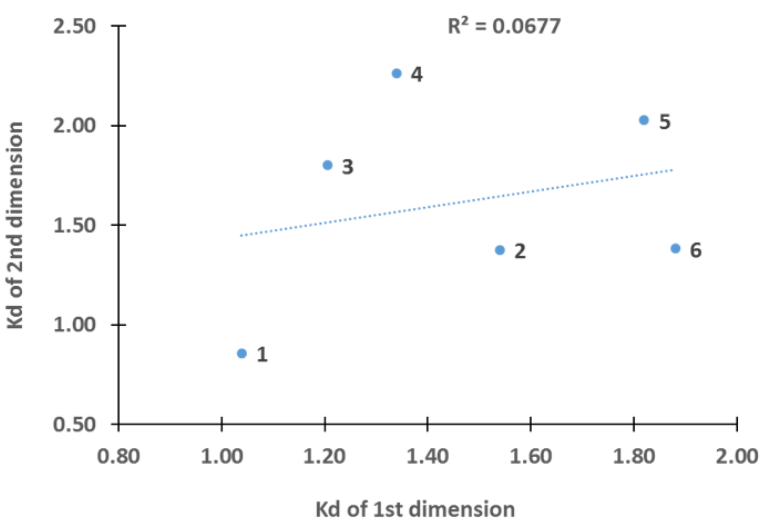

955 Figure 8. 2D orthogonality representations two dimensional LLC-LLC separations in offline mode: (a) $956{ }^{1} D$ solvent system: $n$-hexane/ethyl acetate/methanol/water 3:5:3:5 and ${ }^{2} D$ solvent system: ethyl 957 acetate/ $n$-butanol/water 7:3:10, data from [45]; (b) ${ }^{1} \mathrm{D}$ solvent system: petroleum ether/ethyl 958 acetate/methanol/water 1:1:1:1 and ${ }^{2} \mathrm{D}$ solvent system: petroleum ether/ethyl acetate/methanol/water+Cu${ }^{2+}$ 1:1:1:1, data from [46]. 


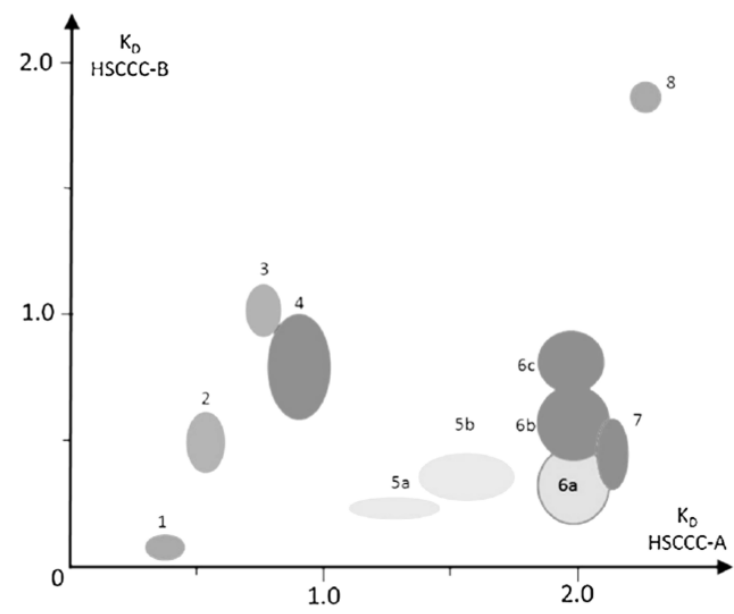

Figure 9. Experimental 2D-plot implemented in das Neves Costa et al. solvent system selection approach. Modified from [47].

(a)

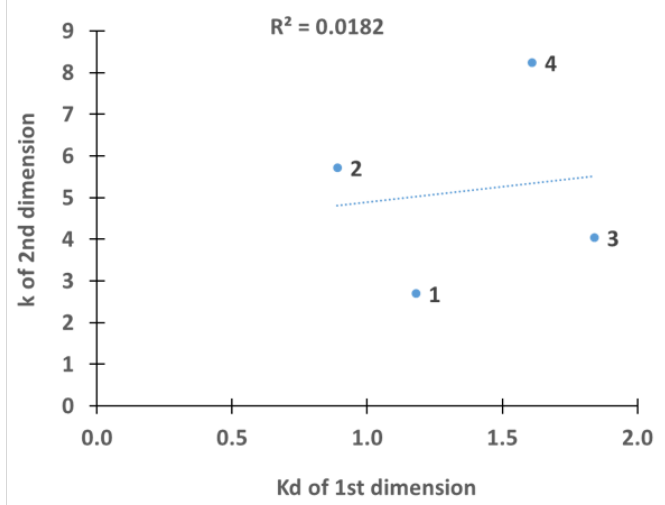

(c)

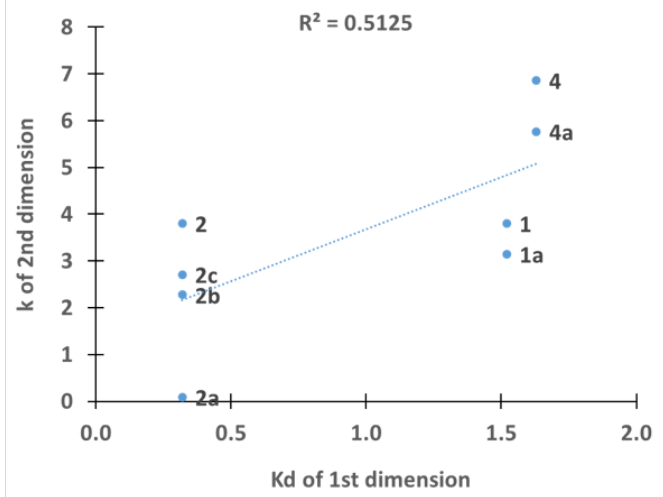

(b)

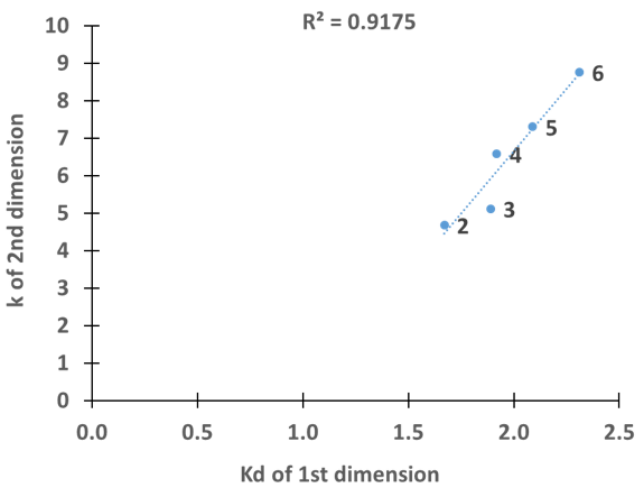

(d)

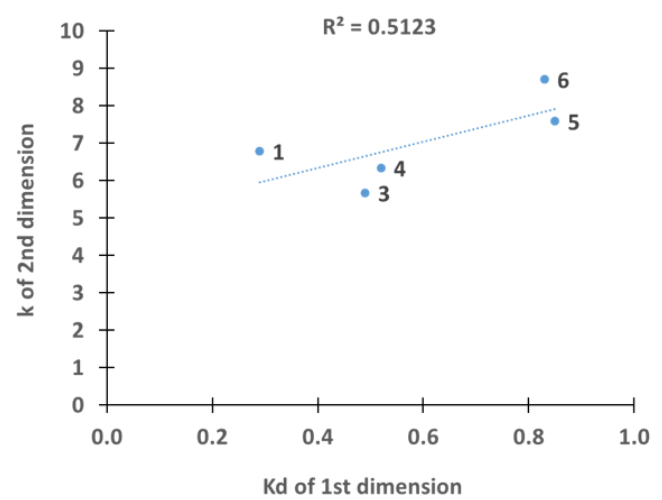

Figure 10. 2D orthogonality representations of offline LLC-HPLC two dimensional separations: (a) ${ }^{1} D$ solvent system: $n$-hexane/ethyl acetate/methanol/water $6: 4: 5: 5$ and ${ }^{2} D$ system: $C 18$ in methanol/water 60:40 isocratic mobile phase; Data from Zhu et al. [49]: (b) ${ }^{1} D$ solvent system: $n$ hexane/ethyl acetate/methanol/water 1:1:1:1 and ${ }^{2} D$ system: ODS in acetonitrile/water 50:50 isocratic mobile phase; Data from Zhu et al. [53]: (c) ${ }^{1}$ D solvent system: methyl ter-butyl ether/ethyl acetate/1butanol/acetonitrile/modified water 1:3:1:1:5 and ${ }^{2} D$ system: ODS in methanol/water isocratic mobile phase; Data from Wang et al. [60] and (d) ${ }^{1} D$ solvent system: ethyl acetate/n-butanol/water 0.5:4.5:5 and ${ }^{2} \mathrm{D}$ system: $\mathrm{C} 18$ in methanol/water isocratic mobile phase; Data from Chen et al. [57] 


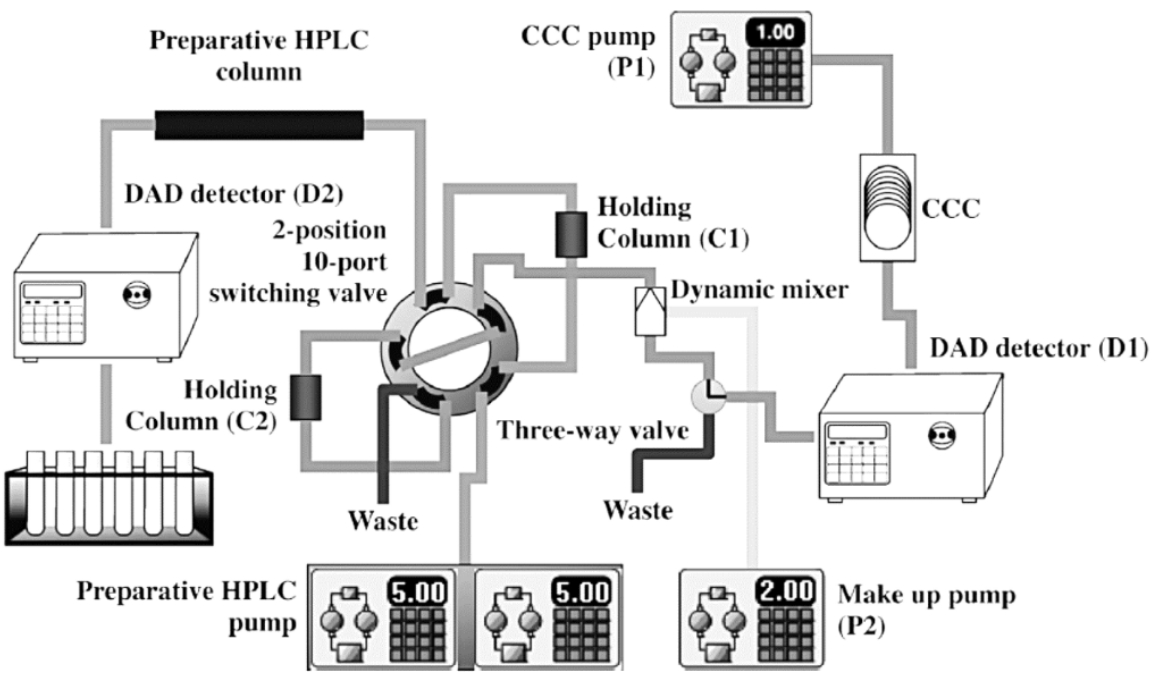

975 Figure 11. Solid-phase trapping columns interface between $1^{\text {st }}$ dimensional LLC and $2^{\text {nd }}$ dimensional HPLC. Modified from [65].

977
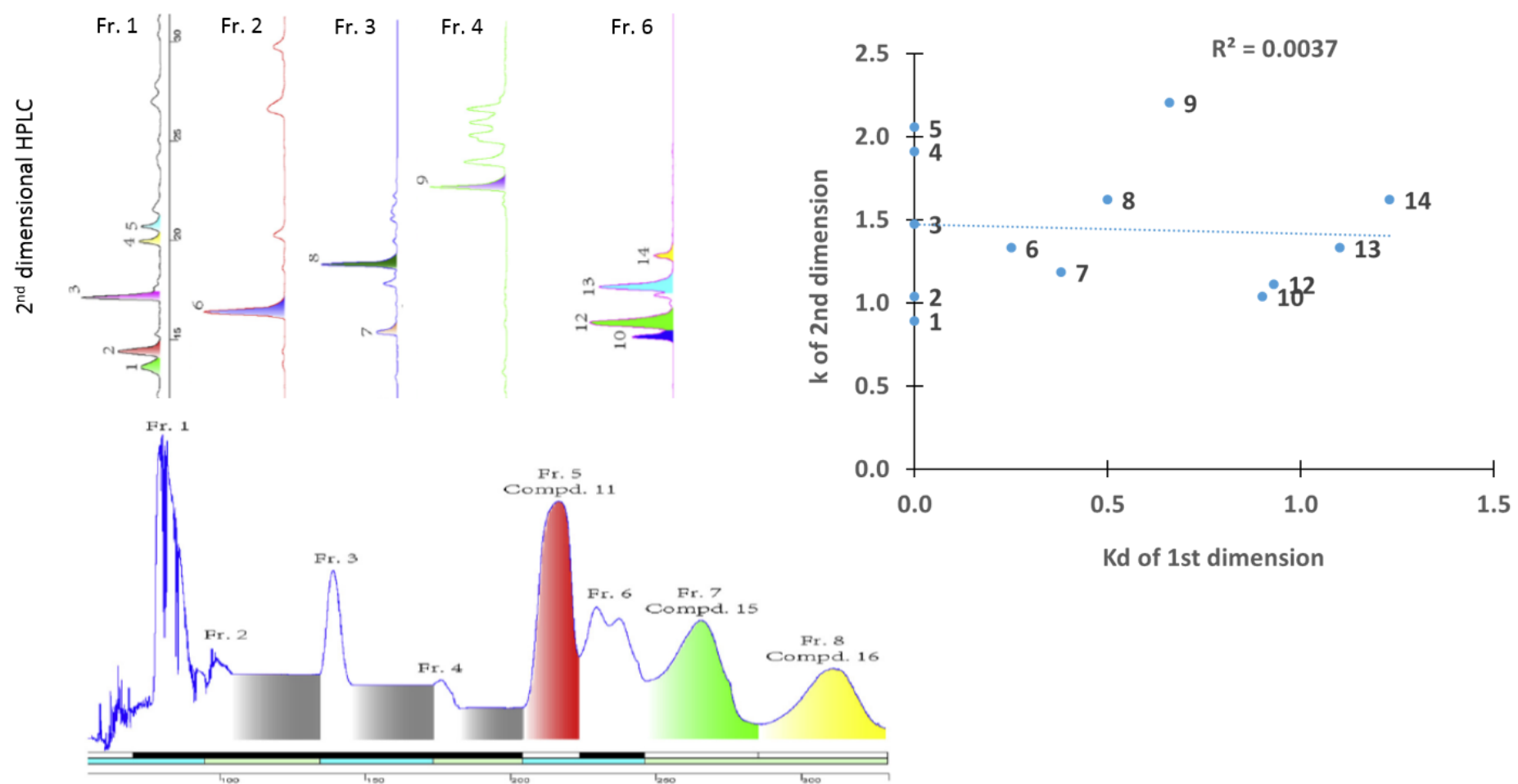

$1^{\text {st }}$ dimensional CCC

Figure 12. Two dimensional LLC-HPLC separation using solid-phase trapping columns interface and stop-and-go mode [66] and its corresponding 2D orthogonality representation: ${ }^{1} \mathrm{D}$ solvent system: $\mathrm{n}$ heptane/acetone/water 31:50:19 and ${ }^{2} \mathrm{D}$ system: $\mathrm{C} 18$ in methanol/water gradient as mobile phase. 